\title{
A novel method based on Adaptive Periodic Segment Matrix and Singular Value Decomposition for removing EMG artifact in ECG signal
}

xieqi chen ( $\nabla$ shayshe@msn.cn )

Southwest Jiaotong University https://orcid.org/0000-0002-9176-2531

Jianhui Lin

Southwest Jiaotong University

Chenguang Huang

Weicai Power CO.,LTd

Yan Huang

Southwest Jiaotong University

Liu He

Southwest Jiaotong University

\section{Research}

Keywords: ECG signal, EMG artifact, Adaptive Periodic Segment Matrix, Singular Value Decomposition, Periodic Segment Estimation Method

Posted Date: December 19th, 2019

DOl: https://doi.org/10.21203/rs.2.19296/v1

License: (1) This work is licensed under a Creative Commons Attribution 4.0 International License. Read Full License

Version of Record: A version of this preprint was published at Biomedical Signal Processing and Control on September 1st, 2020. See the published version at https://doi.org/10.1016/j.bspc.2020.102060. 


\title{
A novel method based on Adaptive Periodic Segment Matrix and Singular Value Decomposition for removing EMG artifact in ECG signal
}

\author{
Xieqi Chen ${ }^{1 *}$, Jianhui Lin ${ }^{1}$, Chenguang Huang ${ }^{2}$, Yan Huang ${ }^{1}$ and Liu $\mathrm{He}^{1}$
}

\footnotetext{
${ }^{*}$ Correspondence: shayshe@msn.cn

${ }^{1}$ State key laboratory of traction power, Southwest Jiaotong University, No. 111, North 1st Section, 2nd Ring Road, Chengdu China

Full list of author information is available at the end of the article

${ }^{\dagger}$ Equal contributor
}

\begin{abstract}
Background: The Electrocardiogram (ECG) signals are usually used to detect and monitor human health. However, the Electromyogram (EMG) artifact also can be obtained during measurement, these make difficult for doctors in correct diagnosis. In general, the ECG signal is periodic while EMG artifact is non-stationary and has overlapped with it under the frequency domain. According to these characteristics, it is necessary to extract clean ECG signals from noisy EMG artifact signals by using the periodic separation method.
\end{abstract}

Methods: A novel Adaptive Periodic Segment Matrix (APSM) based on Singular Value Decomposition (SVD) is proposed for extracting clean ECG from EMG artifact. Firstly a periodic segment estimation method is proposed by obtaining an average periodic length and RR intervals constraint via envelope spectrum of the measured signal. Secondly, the R wave peaks and its position of the ECG signals are detected by these. After that, APSM with rank one is formed using $\mathrm{R}$ wave peaks and the calculated RR intervals constraint, Then SVD is processed on this matrix, the restructured ECG signals will be obtained by the first maximum singular value of the formed matrix. The validation of proposed method is made by applying the algorithm to ECG records from the MIT-BIH Arrhythmia Database. The zero-mean percent root-mean-square difference $\left(P R D_{1}\right)$, Cross-correlation coefficient and output signal to noise ratio $\left(\mathrm{SNR}_{\text {output }}\right)$ have been calculated for presenting the algorithm performance by comparison with other methods. Finally two heart disease cases have been studied for $\mathrm{P}$ wave and ST segment detection under noisy ECG with EMG artifact.

Results: The proposed methods achieved significant improvement in output signal-to-noise ratio, percentage root-mean-square differences and lead to the higher value of cross-correlation coefficient between the original (clean) ECG and the denoised ECG signal. Also, the reconstructed ECG signal can be better able to follow the trend of original (clean) ECG signal under the EMG noise.

Conclusion: The proposed periodic segment estimation method can adaptively find the periodic length in ECG signal by using envelope spectrum. Also, the more strict rank one trajectory matrix has been formed in APSM by using R wave peaks and RR intervals constraint. The results show that the proposed APSM-SVD method is effective for EMG artifact removal and extracting the clean ECG signal. The R peak, P wave, QRS complex and ST segment can be preserved in the reconstructed ECG signal.

Keywords: ECG signal; EMG artifact; Adaptive Periodic Segment Matrix; Singular Value Decomposition; Periodic Segment Estimation Method 


\section{Background}

The Electrocardiogram (ECG) is one of the most important biomedical signals for indicating human health. It provides information about the functional conditions of the heart. The heart rates could partly represent the human body environment and the performances in scenarios[1]. The ECG signals are normally distorted by artifacts that affect the results of health diagnose and monitoring. Therefore, signal denoising and separation have become an essential process in engineering communities[2]. Generally, various artifacts and noise types during ECG recording can lead to erroneous diagnoses. The measured signal contains the artifacts like electrode contact noise, muscle contraction interference, baseline wander, and some instrumental noise generated by the ECG collecting device[3]. All of these can corrupt the ECG and lead to a wrong diagnosis and identification. Statistical techniques such as Principal Component Analysis[4], Independent Component Analysis[5], and Neural Networks[6] have also been used to extract a relatively noise-free signal from noisy ECG. However, those techniques cannot reduce some specific noise in ECG signal.

Many signal processing methods have been used for solving the particular noise. The baseline wander noise arises due to the patient respiration that fluctuates the baseline from zero potential, and lies in the very low-frequency range. The useful frequency range of ECG signal is about 0.5-45 Hz[7]. Therefore, several methods based on the filters can be applied for removing the baseline wander. For example, the high-pass filter[8], nonlinear filter bank[9], median filter and morphology adaptive filter[10], adaptive switching mean filter (ASMF)[11]. Also, other signal processing methods like eigenvalue decomposition[12], Modified Variational Mode Decomposition[13], wavelet transform[15], and Empirical Mode Decomposition[14] can still realize the removal of baseline wander by decomposing the baseline wander and other ECG signals into different components. Powerline (AC) interference is a high-frequency additive noise of 50 or $60 \mathrm{~Hz}$. It is typically a sinusoidal wave with the random phase but constant frequency[15]. This kind of noise comes from the power line to data acquisition device and is present even if special care is taken in proper grounding, shielding, and design of amplifier[16]. It is often removed by a fixed or adaptive notch filter[17]. Hilbert Huang Transform is also used for powerline interference removal[18]. Apart from these, like EMD, eigenvalue decomposition and Modified Variational Mode Decomposition can still remove the powerline interference by decomposing the powerline interference into a single signal component.

Unlike power line interference and baseline wander, the EMG artifact is difficult to detect and eliminate using linear filtering, because of the non-stationary nature of this noise and the big overlaps on whole frequency bands of ECG and EMG signals[19][20]. Due to the characteristics of EMG artifact, several methods based on non-linear filters have been widely used have been applied to denoise the EMG noise to get a clean ECG signal[21], Those method can denoise the EMG artifact but may cause distortion of the ECG signal due to the overlapping with the spectrum[22]. Apart from those filter methods, several scholars have devoted and focused on denoising the ECG signal by using the modern signal processing method. A method based on the wavelet transform was proposed to remove the EMG noise from the ECG signals[23]. Besides, the noisy ECG signal can be cleaned by using Empirical Mode Decomposition (EMD) again[24]. 
Compared with those methods, when applying the wavelet transform method on ECG signals, the selection of the mother wavelet is an unavoidable issue, it often depends on the ECG signal types[25]. For EMD method, there exits the mode mixing and the EMG artifact will be distributed over a number of intrinsic mode functions (IMFs) in the EMD method. Among those existing denoising and separation approaches, the singular value decomposition (SVD) is a method to separate the signal of interest from various noises effectively[26]. A hybrid ECG compression method based on SVD and discrete wavelet transform have been used to extract the ECG from mixing noise[27]. The ECG signal has the characteristic of periodic component[28]. A method based on periodic trajectory matrix and SVD has been applied to extract the fetal ECG from maternal ECG signals[29]. Later, The periodic segment matrix (PSM) has been come out and applied with SVD for detecting and extracting the periodic impulse component in vibration signals[30]. The embedding dimension of periodic segment matrix can utilize singular value ratio (SVR) spectrum and the effective rank order of singular value is equal to one[31]. In fact, due to the random disturbance of EMG artifact, the detected intervals among $\mathrm{R}$ wave peaks fluctuate. At this time, the ECG signal is a pseudo cyclostationary signal, and the traditional SVR spectrum will not be able to determine the embedding dimension. Hence, it is necessary to come out an adaptive periodic segment matrix for extracting clean ECG from EMG artifact.

In this paper, an Adaptive Periodic Segment Matrix (APSM) based on Singular Value Decomposition is proposed to separate the EMG artifact and ECG signals. The average periodic length is firstly calculated by the maximum of envelope spectrum in input signal, also the RR intervals constraint for $\mathrm{R}$ wave peaks selection can be work out. Then find out the $\mathrm{R}$ wave peaks and its positions of the ECG signals by these. After the previous stage, the Adaptive Periodic Segment Matrix can be constructed by trajectory matrix with $\mathrm{R}$ peaks and RR intervals constraint. Singular Value Decomposition is processed on this matrix to work out the first maximum singular value. According to this value, the ECG signals can be reconstructed.

The organization of this paper is as follows. In method section, the theoretical background is provided, the Periodic Segment Matrix (PSM) and the proposed Adaptive Periodic Segment Matrix (APSM) are introduced, the RR intervals constraint and the embedding dimension of proposed matrix is also discussed. Then, the process of proposed method in ECG is presented. The result section indicates the validation of the proposed method by using the MIT-BIH Arrhythmia Database[32]. Also, a comparative analysis of the existing method and the proposed method is carried out in output signal to noise ratio $\left(\mathrm{SNR}_{\text {output }}\right)$, zero-mean percent rootmean-square difference $\left(P R D_{1}\right)$, and cross-correlation coefficient. Finally, in discussion section, the performance of the reconstructed signal in proposed method is compared and discussed with other methods on the time domain waveform. At the same time, two heart disease cases are also analyzed in the P wave and ST segment to show performance of proposed method in doctor diagnosis.

\section{Results}

Simulated signals

In this section, the simulation signals for several different cases are carried out for evaluation and validation of the proposed method. The simulated noisy signal is 
combined with a clean ECG and EMG artifact. The simulated signals can be shown in Eq.1.

$$
s_{\text {Simulated }}=s_{\mathrm{ECG}}+s_{\mathrm{EMG}}
$$

where $s_{\mathrm{ECG}}$ is the clean ECG signal used from the MIT-BIH arrhythmia database[32]. $s_{\text {EMG }}$ is the EMG noise chosen by the muscle (EMG) artifact (in record 'ma') from MIT-BIH Noise Stress Test database[37]. The Figure 1 shows the constructed signals based on the 103 record in MIT-BIH arrhythmia database. Every data file in the database consists of two lead recordings with sampling frequency at $360 \mathrm{~Hz}$ with 11 bits per sample of resolution. The simulation experiment is performed over the EMG noise.
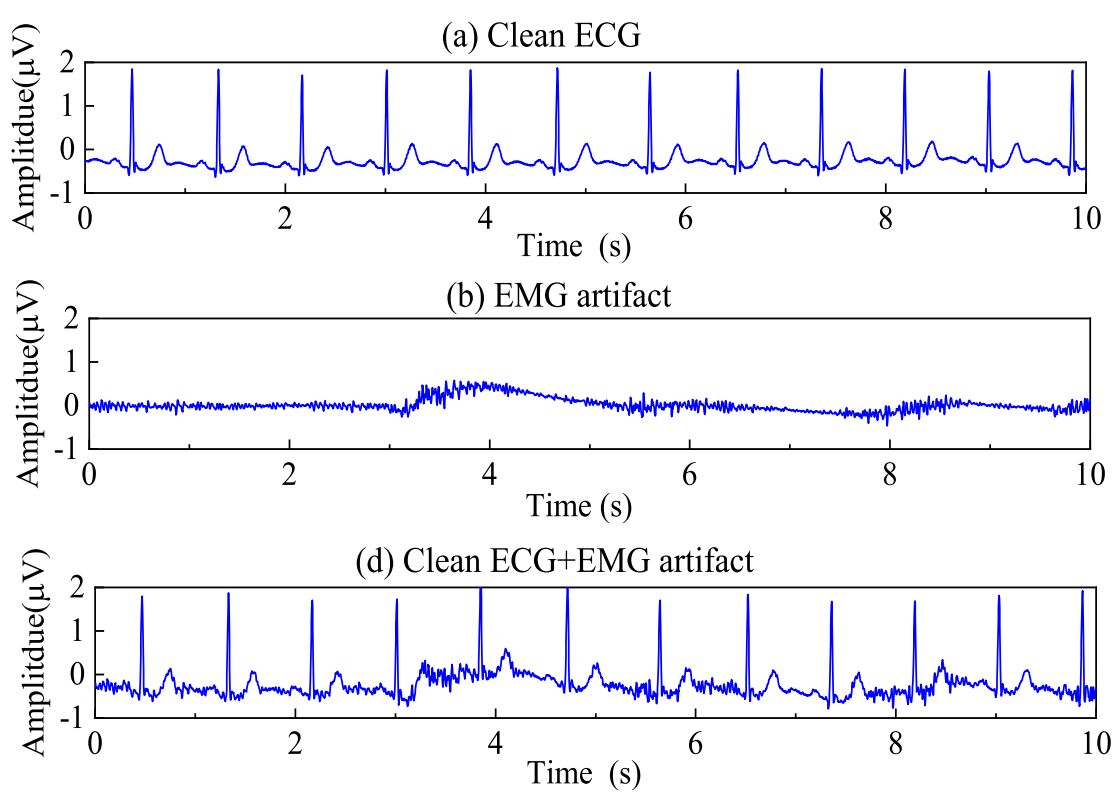

Figure 1 The constructed simulated signals with clean ECG and EMG artifact (a)Clean ECG (b)EMG artifact (c)Clean ECG+EMG artifact

\section{Validation studies in EMG artifact cases}

In this sub-section, the EMG artifact in ECG signals will be discussed. The proposed APSM-SVD method will be used for obtaining the clean ECG signals from EMG artifact, the comparison with EEMD, DWT, PSM-SVD and SSA will be done, and the qualitative and quantitative evaluations will be given and presented. The quantitative evaluation will be progressed with different input/output Signal to Noise ratio $\left(\mathrm{SNR}_{\text {input }}\right)\left(\mathrm{SNR}_{\text {output }}\right)$ and the modified percentage root-mean-square differences $P R D_{1}$ and Cross-correlation coefficient[38]. The Output Signal to Noise ratio $\left(\mathrm{SNR}_{\text {output }}\right)$ will be used for representations and defined as:

$$
\mathrm{SNR}_{\text {output }}=10 \log _{10}\left(\frac{\frac{1}{L} \sum_{l=1}^{L}(\hat{y}[l])^{2}}{\frac{1}{L} \sum_{l=1}^{L}(\hat{y}[l]-y[l])^{2}}\right)
$$


where $y[l]$ and $\hat{y}[l]$ represent original clean ECG signal and reconstructed ECG signal, respectively. In most ECG compression algorithms, the percentage rootmean-square differences $(P R D)$ measure is employed[13] and it can be defined as:

$$
P R D=\sqrt{\frac{\sum_{n=1}^{N}(x(n)-\widehat{x}(n))^{2}}{\sum_{n=1}^{N} x^{2}(n)}}
$$

This error estimate is the one most commonly used in all scientific literature concerned with ECG compression techniques. The clinical acceptability of the reconstructed signal is desired to be as high as possible. The main drawbacks are the inability to cope with baseline fluctuations and the inability to discriminate between the diagnostic portions of an ECG curve. However, its simplicity and relative accuracy that make it a popular error estimate among researchers. As the $P R D$ is heavily dependent on the mean value, it is more appropriate to use the modified criteria. Therefore, the $P R D_{1}$ is proposed for accuracy evaluation in ECG compression. The equation of $P R D_{1}$ has been shown below:

$$
P R D_{1}=\sqrt{\frac{\sum_{n=1}^{N}(x(n)-\widehat{x}(n))^{2}}{\sum_{n=1}^{N}(x(n)-\bar{x})^{2}}}
$$

where the $\widehat{x}(n)$ is the reconstructed signal, and $\bar{x}$ is the mean of the signal. If the $P R D_{1}$ value is between $0 \%$ and $9 \%$, the quality of the reconstructed signal is either 'very good' or 'good'[38], whereas if the value is greater than $9 \%$, its quality group cannot be determined. As we are strictly interested in very good and good reconstructions, it is taken that the value $P R D_{1}$, as measured with Eq.1, must be less than $9 \%$. Hence we compared the performance of our proposed method with three existing techniques: Ensemble Empirical Mode Decomposition, Discrete Wavelet Transform and Singular Spectrum Analysis (SSA). In EEMD method, the noisy ECG signal is decomposed into intrinsic mode functions (IMFs) and the last three IMF are discarded. Discrete Wavelet Transform (DWT) uses filter banks for construction of the multi-resolution analysis with relatively low computation time. The procedure of SSA is usually divided into the following steps: embedding, singular value decomposition, grouping and reconstruction. PSM-SVD method is similar to the proposed APSM-SVD, but the periodic segment is fixed.

Table 1 Comparison of SNR output for the proposed APSM-SVD method with EEMD, DWT, SSA and PSM-SVD averaged over 5 segments

\begin{tabular}{cccccc}
\hline & \multicolumn{5}{c}{ SNR $_{\text {output }}(\mathrm{dB})$} \\
\hline SNR $_{\text {input }}(\mathrm{dB})$ & EEMD & DWT & SSA & PSM-SVD & APSM-SVD \\
-20 & 0.039 & 0.045 & 0.054 & 0.0225 & 0.063 \\
-15 & 0.085 & 0.202 & 0.25 & 0.0556 & 0.329 \\
-10 & 0.133 & 0.478 & 0.61 & 0.1043 & 0.683 \\
-5 & 0.376 & 1.139 & 1.582 & 0.5079 & 1.749 \\
0 & 1.217 & 4.321 & 9.402 & 6.8342 & 12.421 \\
\hline
\end{tabular}

It is evident from Table.1 and Fig.2 that $\mathrm{SNR}_{\text {output }}$ obtained by all the methods are larger than those in $\mathrm{SNR}_{\text {input }}$. Meanwhile, the proposed method has relatively higher output signal to noise ratio than other methods under the same level. As 


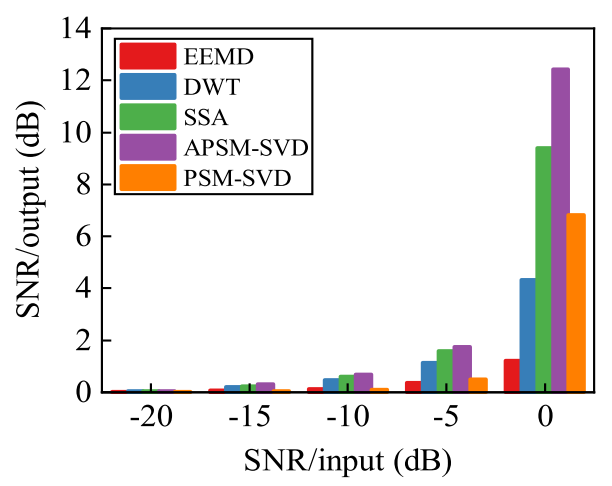

Figure 2 The comparative performance SNR output of the proposed APSM-SVD with other methods over 5 segments

Table 2 Comparison of cross-correlation coefficient for the proposed APSM-SVD method with EEMD, DWT, PSM-SVD and SSA averaged over 5 segments

\begin{tabular}{ccccccc}
\hline \multicolumn{7}{c}{ Cross-correlation coefficient } \\
\hline SNR $_{\text {input }}(\mathrm{dB})$ & Input noisy signal & EEMD & DWT & SSA & PSM-SVD & APSM-SVD \\
-20 & 0.053 & 0.051 & 0.039 & 0.053 & 0.0227 & 0.055 \\
-15 & 0.123 & 0.144 & 0.093 & 0.123 & 0.133 & 0.226 \\
-10 & 0.214 & 0.392 & 0.163 & 0.214 & 0.1043 & 0.631 \\
-5 & 0.449 & 0.662 & 0.343 & 0.447 & 0.134 & 0.929 \\
0 & 0.904 & 0.883 & 0.693 & 0.895 & 0.843 & 0.966 \\
\hline
\end{tabular}

the $\mathrm{SNR}_{\text {input }}$ increase, the obtained $\mathrm{SNR}_{\text {output }}$ increased. Still, the APSM-SVD performs better than the other method. Moreover, the Cross-correlation coefficient is also calculated with clean ECG and shown in Table.2. The Cross-correlation coefficient of input noisy signal is smaller when $\mathrm{SNR}_{\text {input }}$ is lower, which matches the EMG noise is larger under the lower signal to noise ratio.

Nevertheless, the proposed method still achieved better results compared with others in Cross-correlation coefficient. Also, the performance measures for $P R D_{1}$ under different $\mathrm{SNR}_{\text {input }}$ have been calculated and shown in Table.3. The $P R D_{1}$ can be guarantee good reconstructed signals under the range of 0\%-9\%. Particularly, the $P R D_{1}$ exceeds $9 \%$ when $\mathrm{SNR}_{\text {input }}$ is $-20 \mathrm{~dB}$ in EEMD, DWT, SSA and PSM-SVD,

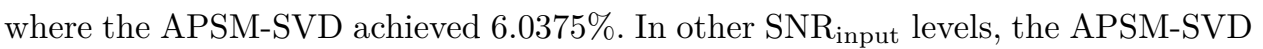
has lower $P R D_{1}$ compared with other methods. Therefore, the proposed APSMSVD would be a promised method for separate clean ECG from EMG artifact.

Table 3 Comparison of the percentage root-mean-square differences among the EEMD, DWT, SSA, PSM-SVD and proposed APSM-SVD

\begin{tabular}{cccccc}
\hline \multicolumn{6}{c}{ Performance measures PRD $(\%)$} \\
\hline SNR $_{\text {input }}$ & EEMD & DWT & SSA & PSM-SVD & APSM-SVD \\
-20 & 12.075 & 13.673 & 13.56 & 13.66 & 6.0375 \\
-15 & 5.156 & 7.052 & 6.963 & 6.88 & 4.159 \\
-10 & 4.023 & 4.255 & 4.175 & 4.19 & 3.018 \\
-5 & 1.41 & 2.083 & 1.957 & 2.07 & 1.217 \\
0 & 0.795 & 0.826 & 0.476 & 0.553 & 0.156 \\
\hline
\end{tabular}

Different records may have different denoised performance, the performance measures for $P R D_{1}$ in different MIT-HIH records have been calculated and shown in Table.4, as the $P R D_{1}$ decreases, the better the performance is, this also obeys the 
rule with good reconstructed signals under the range of 0\%-9\%. Therefore, the results show that the APSM-SVD achieve better performance compared with other method among the records.

Table 4 Comparison of the percentage root-mean-square differences among the EEMD, DWT, SSA, PSM-SVD and proposed APSM-SVD in different MIT-BIH records

\begin{tabular}{cccccc}
\hline \multicolumn{5}{c}{ Performance measures $P R D_{1}(\%)$} \\
\hline MIT-BIH record & EEMD & DWT & SSA & PSM-SVD & APMSVD \\
103dat & 0.795 & 0.826 & 0.476 & 0.553 & 0.156 \\
104dat & 1.021 & 0.739 & 0.853 & 0.563 & 0.522 \\
105dat & 0.889 & 0.624 & 0.515 & 0.502 & 0.269 \\
117dat & 3.655 & 0.792 & 0.669 & 0.768 & 0.465 \\
118dat & 2.221 & 0.584 & 0.389 & 0.407 & 0.187 \\
119dat & 1.806 & 0.488 & 0.632 & 0.333 & 0.314 \\
214dat & 0.831 & 0.472 & 0.413 & 0.375 & 0.352 \\
231dat & 0.892 & 0.831 & 0.475 & 0.590 & 0.310 \\
\hline
\end{tabular}

\section{Discussion}

In order to better reflect the effect of noise reduction, the time domain waveform after denoised and separated of the proposed method and other methods are also shown in Fig.3. The reconstructed signals in EMD, DWT, SSA and PSM-SVD method still have some EMG artifact compared with the clean ECG signal in 103 record . However, the reconstructed ECG signal in proposed APSM-SVD method is better and can quite follow the clean ECG signal.

In many cases, we need to recognize the R peak of the original (clean) ECG along with the $\mathrm{P}$ wave, T wave, QRS complex and the ST segment for correct heart disease identification. It can be seen from Fig.4 (a) and (b) that all the methods can identify the $\mathrm{R}$ peak. There is a small phase shift in DWT results, due to the addition of the EMG artifact, the noisy ECG signal has a baseline shift compared with clean ECG signal. This causes the baselines of reconstructed results in DWT and SSA are similar to the noisy ECG signal, and cannot detect the actual baseline of the clean ECG signal.The reconstructed signal in PSM-SVD also has a phase shift compared with APSM-SVD.But the effect of baseline shift is better than other methods. Meanwhile, EEMD method can quite detect the baseline of clean ECG. But there still existed the incorrect identification in $\mathrm{P}$ wave and $\mathrm{T}$ wave. The proposed APSMSVD is better able to follow the original (clean) ECG signal under the EMG noise. Also, the R peak, QRS complex and the ST segment can be detected correctly in comparison to the other methods.

General speaking, in ECG signal, R peak and QRS complex of ECG signal has periodicity, and then EMG signal is random with no periodicity. Therefore, the Adaptive Periodic Segment Matrix with rank one is formed by periodic segment of noisy ECG signal, and then process to singular value decomposition for reconstructing the clean ECG signal. The left singular value matrix is a single waveform of QRS complex, and the right singular value matrix is the coefficient, so as to reconstruct a new ECG signal. Meanwhile, EEMD does not have the ability to extract the two signals in the similar frequency band, but for DWT, the result of DWT method depends on the similarity between wavelet base and target signal. In reality, it is often difficult to achieve. At the same time, the quality of SSA depends on the segment length of trajectory matrix, it is difficult to determine a suitable segment 

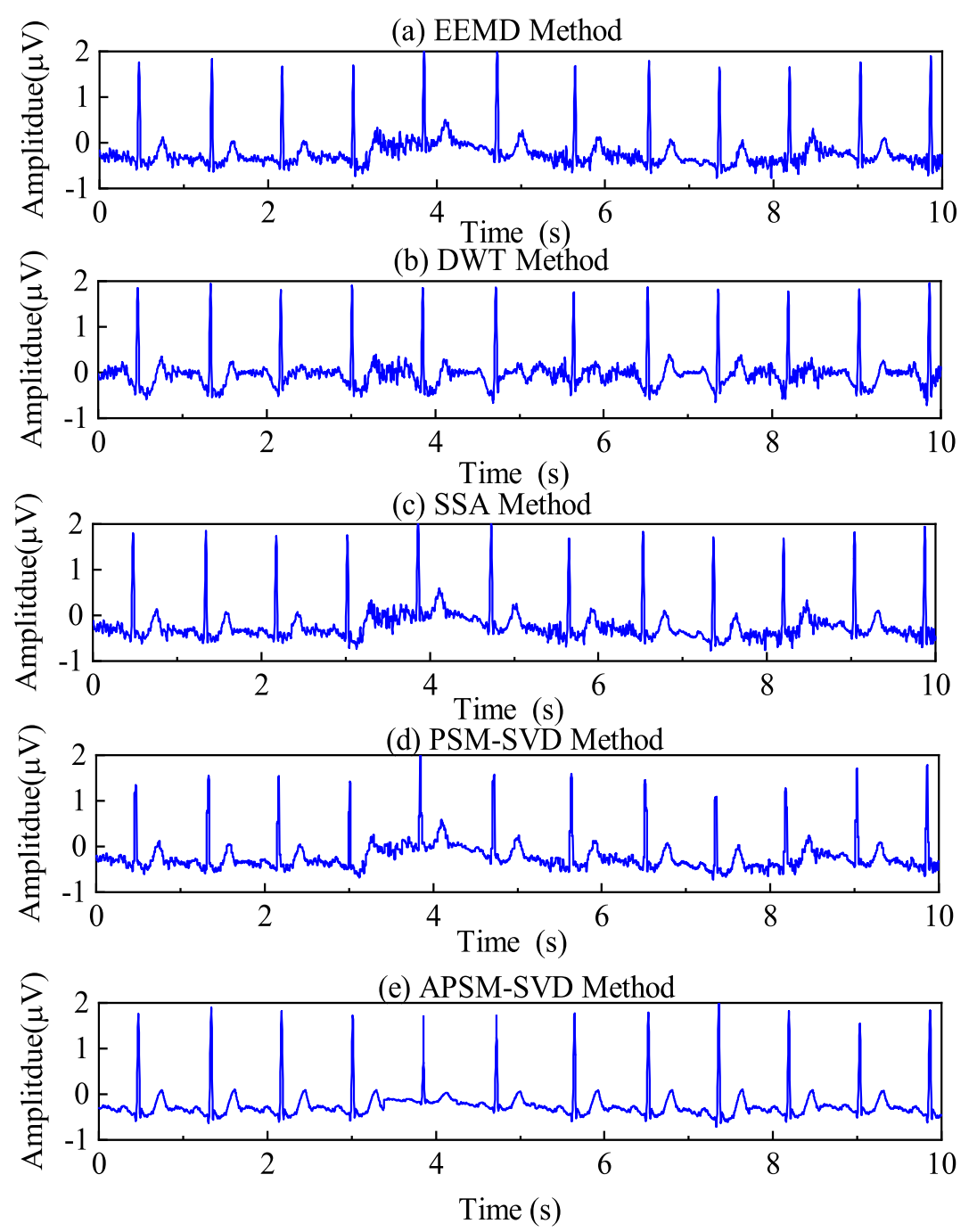

Figure 3 Reconstructed ECG signals from noisy ECG record by using the proposed APSM-SVD, EEMD, DWT, PSM-SVD and SSA(a)EEMD (b)DWT (c)SSA (d)PSM-SVD (e)APSM-SVD

length without prior knowledge. The core innovation of this paper is to provide a suitable period segment estimation method and ensure the formed trajectory matrix is strict rank one matrix by average period length in envelop spectrum and RR intervals constraint in $\mathrm{R}$ wave peak selection. Also the Periodic Segment Matrix has embedding dimension, but it can not be changed as the length of signals increases. The proposed APSM-SVD based on this method can separate EMG artifact and ECG signal, where traditional methods cannot solve this problem. 


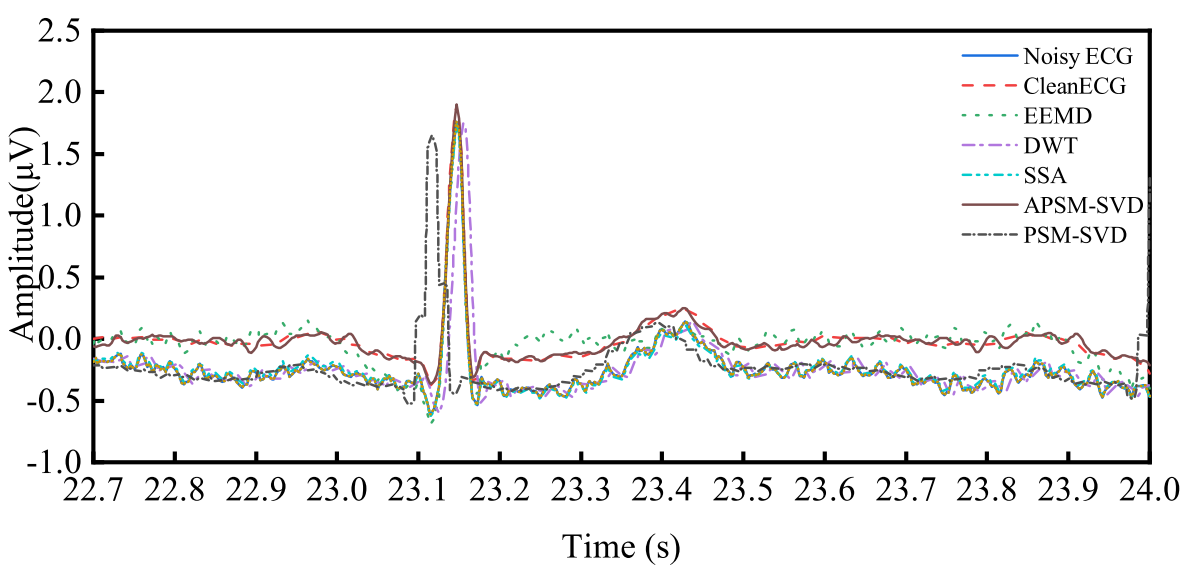

(a) The amplitude of EMG noise is relatively small

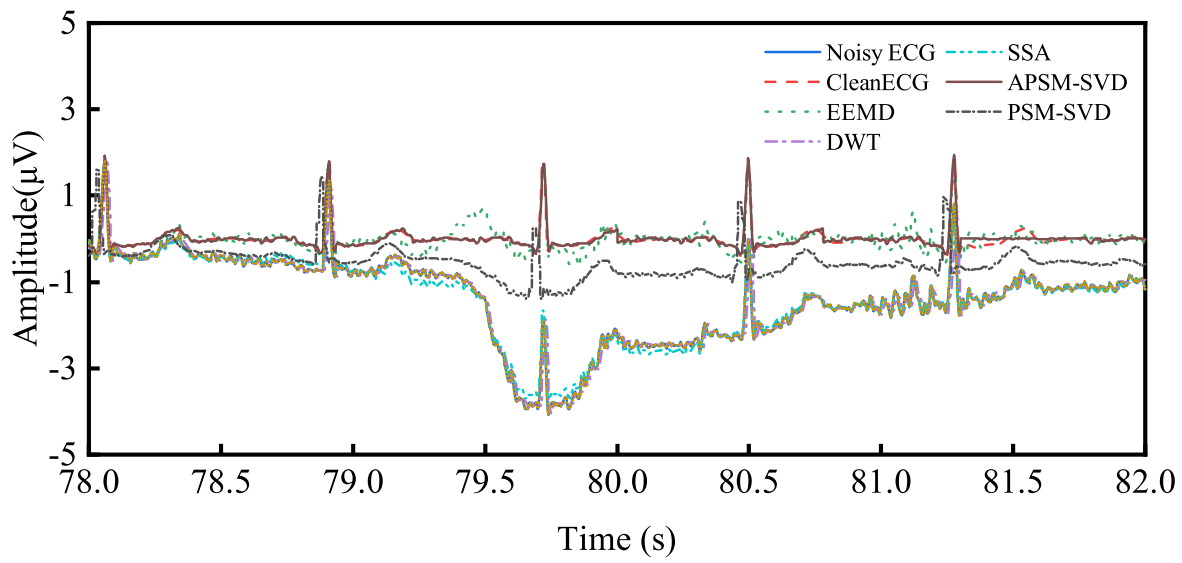

(b) The amplitude of EMG noise is relatively larger

Figure 4 The reconstructed signals obtained from the proposed technique compared to EMD, DWT, SSA and PSM-SVD for QRS complex in 103 record.(a) The amplitude of EMG noise is smaller (b)The amplitude of EMG noise is larger

The application in heart disease identification

Left bundle branch block (LBBB) case studies

In previous section, the validation has been made for proposed method. Therefore, in this section, the application of the proposed method in heart disease is discussed. Hence, the LBBB case has been applied. LBBB is a cardiac abnormality that is mainly caused due to delay in activation of the left ventricle[39]. ECG recordings of patients suffering with LBBB have the following characteristics: (1) QRS duration is greater than $120 \mathrm{~ms}$; (2) Lead V1 signal shows a slurring of QRS with an initial $\mathrm{R}$ wave; (3) ST segment has displacement; (4) the direction of $\mathrm{T}$ wave is opposite to $\mathrm{R}$ wave. The Record 214 of MIT-BIH Arrythmia database shows ECG signal with LBBB. The identification has been shown in Fig.5.

Compared with noisy ECG, the proposed APSM-SVD can denoise the EMG artifact and reconstructed the target ECG. While the PSM-SVD still has EMG artifact in reconstructed signal. The $\mathrm{R}$ waves can be detected and matched with the clean 

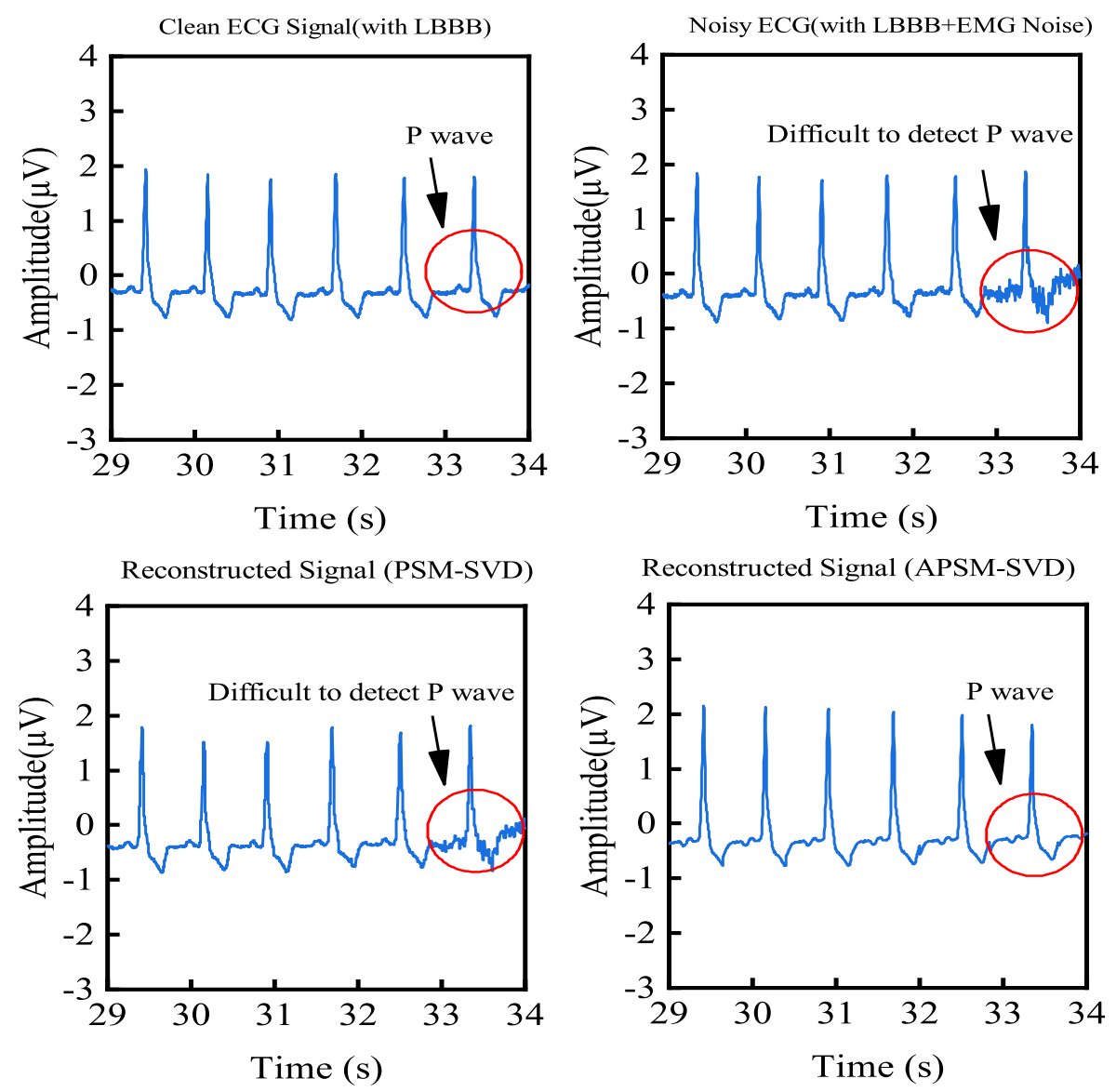

Figure 5 The detection of the R peak, $\mathrm{P}$ wave and QRS complex in 214 LBBB record

ECG by using APSM-SVD. P wave is difficult to identify in noisy ECG signal and reconstructed signal in PSM-SVD. This may generate false alarm for heart abnormality known as atrial fibrillation.

ST elevation myocardial infarction (STEMI) studies

ST segment elevation and depression is used for identification of ST elevation myocardial infarction (STEMI)[40]. STEMI is a type of heart attack in which a coronary artery is blocked completely by a blood clot. Some heart muscles which receive oxygen from that coronary artery begin to die. The highly elevated ST segment indicates the amount of heart muscle damage. We know that EMG noise affects the ST segment. However, for disease identification such as STEMI, it is necessary to preserve the ST segment in the denoised signal. To test the efficacy of our proposed method, we use Record 231 with straight elevation (characteristic of STEMI) in the ST segment (Fig.6). Noisy ECG signal is created by adding EMG noise to the clean ECG signal. And the reconstructed signals are obtained by the proposed APSMSVD and PSM-SVD. It can be seen that in Fig.6, although the $\mathrm{R}$ wave peaks can be detected correctly in all signals, the EMG artifact in noisy ECG seriously affects ST elevation recognition. On denoising with proposed method, we notice that 

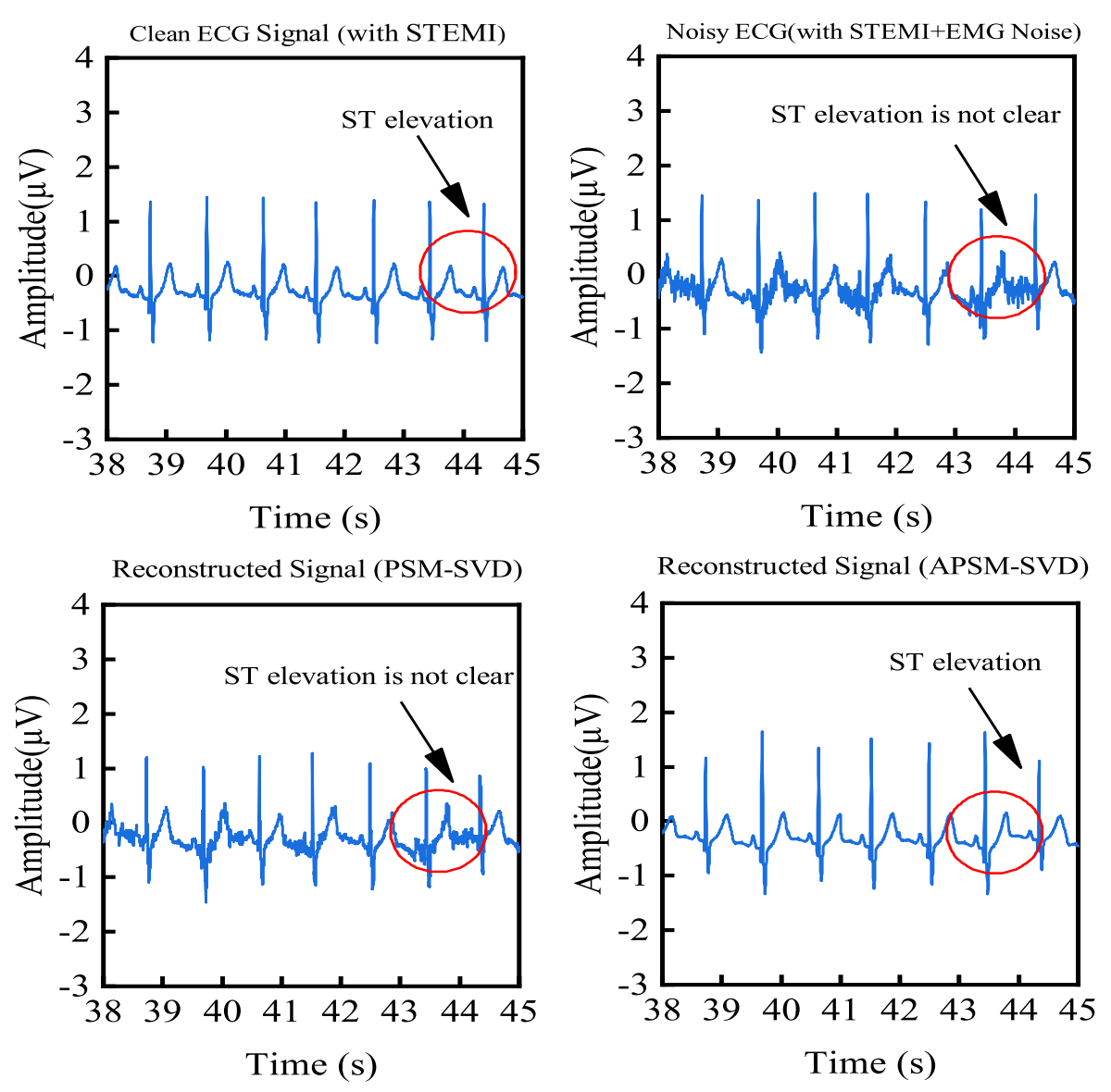

Figure 6 The detection of the R peak, ST elevation and QRS complex in 231 STEMI record

the straight elevation of ST segment is restored and STEMI is correctly detected. Where that in PSM-SVD is not clear. Therefore, the alarm for atrial myocardial infarction can be applied by the proposed method. 


\section{Conclusion}

Real-time ECG signals are often suffered from the EMG artifact that need to be denoise and removed before an ECG signal can be used by a doctor for analysis. The ECG signal has the periodic components and EMG is nature random, Therefore, the denoised method based on the periodic segments can extract the clean ECG signal. However, the periodic segments in measured ECG signal will affect the performance of the reconstruction. And the periodic segments will be changed due to the different types of ECG. In this paper, a new periodic segment estimation method and an Adaptive Periodic Segment Matrix based on SVD has been proposed to extract the clean ECG signal in noisy ECG signal with EMG artifact. Compared with Periodic Segment Matrix, the envelop spectrum is used for calculating the average period length. Then RR intervals constraint has been made for selecting the $\mathrm{R}$ wave peaks. Also, the embedding dimension is adaptively selected from this. Still, the adaptive periodic segmented matrix is constructed by RR intervals constraint from a $\mathrm{R}$ wave peak to both sides, so as to ensure the formation of more strict rank one matrix in different ECG signals. The validations of the proposed method have been made and the comparison also has been done. Performance comparison shows that, compared to other methods, the proposed methods provide significant improvement in output signal-to-noise ratio, percentage root-mean-square differences and lead to higher value of cross-correlation coefficient between the original (clean) ECG and the denoised ECG signal. The time domain waveform after denoised is also compared. The reconstructed signal in proposed APSM-SVD can follow the clean ECG signal and the $\mathrm{P}$ wave, QRS complex, ST segment and $\mathrm{R}$ wave can be preserved. EMG artifact affect the ST segment and small amplitude waves i.e. $\mathrm{P}$ wave and the ST segment of the clean diseased (original) ECG signal. Thus, the two heart disease cases (LBBB) and(STEMI) are also applied in PSM-SVD and APSM-SVD method. The results show that $\mathrm{P}$ waves and ST segment can be preserved in APSM-SVD. Therefore, Adaptive Periodic Segment Matrix can be used to form the trajectory matrix and process to SVD for restructuring the pure ECG to preserve the R peak, $\mathrm{P}$ wave, $\mathrm{T}$ wave and the $\mathrm{ST}$ segment for correct diagnosis. We have also demonstrated that our proposed APSM-SVD method is able to avoid the effect of EMG noise on these. Therefore, the proposed APSM-SVD can perfectly achieve the requirements of extracting clean ECG from noisy ECG signals with EMG artifact. In further, during the measurement condition of wearable device, the amplitude of EMG signals may far exceed in ECG signals, which leading to its peak value cannot be accurately identified, how to extract the pure signal through the proposed method needs further research and discuss.

\section{Method}

Periodic Segment Matrix

Many methods can be used to construct the trajectory matrix. One of the most famous methods is the Hankel matrix[33][34]. However, the Hankel matrix is unsuitable for strengthening the periodic impulse responses. Therefore, the novel trajectory matrix, Periodic Segment Matrix (PSM), without accumulative error[29] is used as the trajectory matrix of SVD. The trajectory matrix with PSM properties 
can be expressed as:

$$
\mathbf{Y}=\left(\begin{array}{ccc}
\mathbf{s}\left(c_{1}+1\right) \mathbf{s}\left(c_{1}+2\right) & \ldots & \mathbf{s}\left(c_{1}+l\right) \\
\mathbf{s}\left(c_{2}+1\right) \mathbf{s}\left(c_{2}+2\right) & \ldots & \mathbf{s}\left(c_{2}+l\right) \\
\vdots & \ddots & \vdots \\
\mathbf{s}\left(c_{a}+1\right) \mathbf{s}\left(c_{a}+2\right) & \cdots & \mathbf{s}\left(c_{a}+l\right)
\end{array}\right)
$$

where $\mathbf{s}$ is any periodic impulse component, $\alpha$ is the number of periods, and $l$ is the embedding dimension. $l=\left\langle p>, c_{i}=\left\langle(i-1) p>, i \in[1, a], l, a \in \mathbf{N}^{*}, l \geq 2, a \geq 2\right.\right.$, and $c_{a}+l \leq \mathbf{N}$, where $\left\langle.>\right.$ is a rounding operator, $p$ is $h$ times $T$, and $h \in \mathbf{N}^{*}$. $T$ is the period length of the periodic impulse component and can be determined by the singular value ratio (SVR) spectrum[31]. Peaks at higher multiples of this length must be monitored. Therefore, the embedding dimension $l$ can be expressed as

$$
l=<p>=<h T>
$$

Naturally, the row number $a, \mathbf{Y}$ is also determined as

$$
a=\operatorname{argmax}<(a-1) h \mathrm{~T}>+<h \mathrm{~T}>
$$

$h$ can be obtained by maximizing the rank of a matrix $\mathbf{Y}$, i.e.,

$$
h=\underset{h}{\operatorname{argmax}} \operatorname{rank}(\mathbf{Y})
$$

Where rank $(\mathbf{Y})$ is the rank of a matrix $\mathbf{Y}$ and is equal to $\min (a, l)$. And this of a pure periodic signal is equal to 1 , the trajectory matrix $\mathbf{Y}$ can be reconstructed by using the first maximal singular value, i.e.

$$
\hat{\mathbf{Y}}=\sigma_{1} \mathbf{u}_{1} \mathbf{v}_{1}^{\mathrm{T}}
$$

Finally, the periodic impact component $\hat{\mathbf{Y}}$ is extracted by the inverse process of $\mathbf{Y}$ in Eq.5.

General speaking, the periodic segment matrix can separate the strictly periodic signals, and it can be extracted by SVD based on the rank of the trajectory matrix equals to one. However, the periodic segment in PSM may not be appropriate for the noisy ECG with the EMG artifact, the peaks in SVR spectrum cannot be able to find. This will cause the rank of the trajectory matrix is not a rank one matrix. The first singular value of this method will not preserve the $\mathrm{P}$ wave, QRS complex, $\mathrm{T}$ wave and ST segment of ECG component. Therefore, the ECG signal will fail to be reconstructed.

\section{Adaptive Periodic Segment Matrix}

As mentioned above, the clean and periodic ECG signal can be detected by using the Periodic Segment Matrix. Once the EMG artifact has been added, the embedding dimension and periodic segment in PSM will not be suitable, even though using the 
particular embedding dimension. In other words, the rank of the matrix will not be equal to one, this affects the signal recovery after processing the SVD. Therefore, according to this shortage in PSM and the requirement for extracting the clean ECG from EMG noise, a new matrix named Adaptive Periodic Segment Matrix is proposed to form a strict rank one matrix. In ECG research field, scholars usually pay more attention to the $\mathrm{P}$ wave, $\mathrm{R}$ wave, ST segment and QRS complex for ECG signals. As a result, the peaks are found and pursuit in the proposed method, the embedding dimension of the matrix has been selected based on the RR intervals constraint. These steps can choose the suitable periodic segment for ECG signal to make the rank of the trajectory matrix strictly equals to one, and guarantee the correct $\mathrm{P}$ wave, $\mathrm{R}$ wave, $\mathrm{ST}$ segment and QRS complex for reconstructed ECG signal.

Peak pursuit

In the ECG signal, $R$ wave peak needs to be recognized, but due to the interference of EMG noise, directly finding $\mathrm{R}$ wave peaks will include some other peaks which not belong to $\mathrm{R}$ wave, so a certain $\mathrm{RR}$ intervals constraint needs to be set when pursuing for correct $\mathrm{R}$ wave peaks. If the selected interval is too large, the $\mathrm{R}$ wave peaks will not be fully recognized. Otherwise, if the interval is too small, the peak value that not belongs to $\mathrm{R}$ wave will be wrongly selected. Therefore, here is an assumption of RR intervals constraint:

$$
\left\lceil\frac{\max \left(R R_{\text {intervals }}\right)}{2}\right\rceil<Z<\left\lceil\min \left(R R_{\text {intervals }}\right)\right\rceil
$$

where $Z$ is the selected $R R$ intervals constraint. In reality, the maximum $R R$ interval and the minimum RR interval are not very clear due to the noise. This makes it difficult to choose the $Z$. As we know, the fundamental frequency of the envelope spectrum reflects the average period of the main components of the signal. Hence, the position corresponding to the maximum value of envelope spectrum can be taken as mean $\left(R R_{\text {intervals }}\right)$. Also, there is little difference between the maximum and minimum RR intervals in normal ECG signals, both of them can be approximated as mean $\left(R R_{\text {intervals }}\right)$, so the above Eq.10 can be written as:

$$
\left\lceil\frac{\text { mean }\left(R R_{\text {intervals }}\right)}{2}\right\rceil<Z<\left\lceil\text { mean }\left(R R_{\text {intervals }}\right)\right\rceil
$$

where mean(.) is the mean value of the $R R_{\text {intervals. }}$ Therefore, the $R R$ intervals constraint $Z$ can be rewritten as:

$$
Z=\left\lceil\alpha \cdot \operatorname{mean}\left(R R_{\text {intervals }}\right)\right\rceil, \alpha=\left(1, \frac{1}{2}\right)
$$

In this paper, we choose $\alpha=2 / 3$ as the coefficient for $Z$. Hence, the $\mathrm{R}$ wave peaks can be found as $\left[R_{1}, \ldots, R_{n}\right]$ and their positions $\left[X_{1}, \ldots, X_{n}\right]$. 
Trajectory matrix construction

In order to ensure the trajectory matrix is a strict rank one matrix. The position matrix $\mathbf{B}$ based on $\mathrm{R}$ wave peak position can be written as:

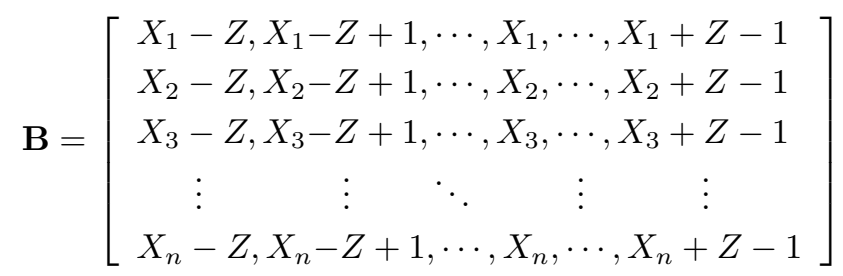

where $Z=\left\lceil\frac{2}{3} \cdot \operatorname{mean}\left(R R_{\text {intervals }}\right)\right\rceil$ Then the trajectory matrix $\mathbf{Y}_{\text {apsm }}$ can be formed

$$
\mathbf{Y}_{\text {apsm }}=\mathbf{s}(\mathbf{B})
$$

Besides, $\mathbf{s}$ are the input signal, $n$ is the number of $\mathrm{R}$ wave peak. The embedding dimension selection here can be noted:

(1) If embedding dimension is smaller than any $R$ wave peak intervals, the reconstructed period ECG component may not cover all QRS complex, P waves and ST segments, which will lose the feature of the ECG signal.

(2) If embedding dimension is bigger than any $\mathrm{R}$ wave peak intervals, the two ends of reconstructed ECG signal will exceed the length of input signal, which cannot reconstruct or reconstruct false ECG components.

Therefore, the embedding dimension in proposed matrix is selected as double length of $Z$ to obtain both requirements above.

For the periodic segment matrix, with the increase of signal length and the decrease of signal stability, every periodic component of its trajectory matrix will shift, and the periodic segment mode will break, which will not form a rank one matrix. Compared with PSM, the trajectory matrix in APSM is traversed from R wave peak to both sides, so the constructed matrix can be a more strict rank one matrix. The comparison of the trajectory matrix in $\operatorname{APSM(Eq.14)~and~PSM~(Eq.5)~}$ has been shown in Fig.7. It can be seen that the detected periodic components in PSM has a shift in different rows. This will make the formed trajectory matrix in PSM will not be a strict rank one matrix. As a result, the first maximum singular value will recover the false ECG signal. 


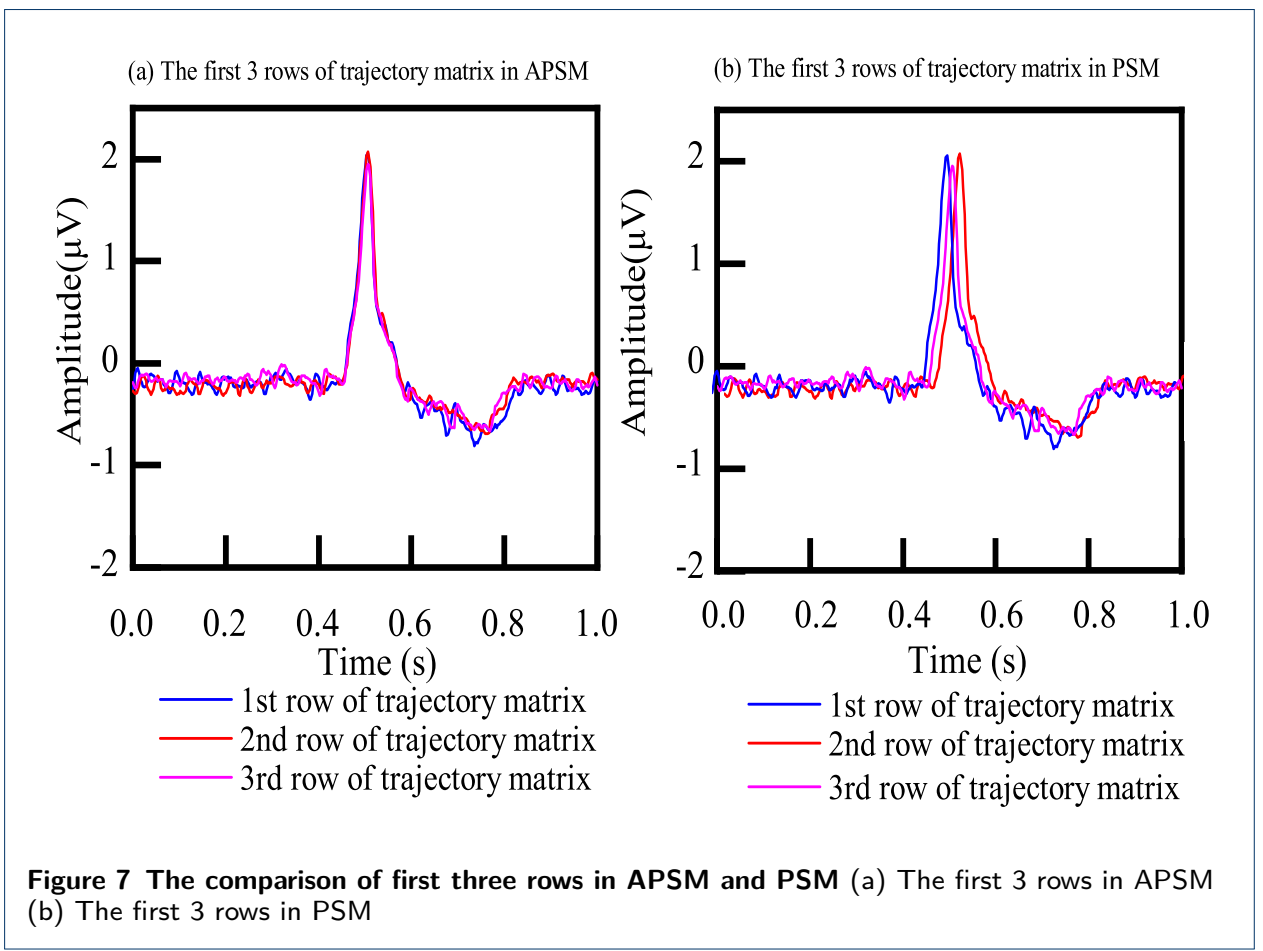

Singular value decomposition

The formed trajectory matrix $\mathbf{Y}_{\text {apsm }}$ would be input to the process of SVD, SVD has been widely utilized as an effective method for enhancing periodic impact components[35][36]. For an arbitrary real $m \times n$ matrix $\mathbf{Y}$, SVD can be expressed as:

$$
\mathbf{Y}_{m \times n}=\mathbf{U}_{m \times m} \mathbf{D}_{m \times n} \mathbf{V}^{\mathrm{T}}{ }_{n \times n}
$$

where $\mathbf{U}_{m \times m}=\left[\mathbf{u}_{1}, \mathbf{u}_{2}, \cdots, \mathbf{u}_{m}\right], \mathbf{V}_{n \times n}=\left[\mathbf{v}_{1}, \mathbf{v}_{2}, \cdots, \mathbf{v}_{n}\right]$, and $\mathbf{D}_{m \times n}=\left[\begin{array}{cc}\mathbf{C}_{q \times q} & \mathbf{O} \\ \mathbf{O} & \mathbf{O}\end{array}\right]$, $\mathbf{C}_{q \times q}=\operatorname{diag}\left(\sigma_{1}, \sigma_{2}, \cdots, \sigma_{q}\right), q=\min (m, n)$, and diagonal elements $\sigma_{i}(i=1, \cdots, q)$ are the singular values of $\mathbf{Y}$, and $\sigma_{1}>\sigma_{2}>\ldots>\sigma_{q}$.

Matrix Reconstruction and Signal Recovery

Similar to the periodic segment matrix reconstruction, the rank of trajectory matrix $\mathbf{Y}_{\text {apsm }}$ is equal to 1, the trajectory matrix $\mathbf{Y}_{\text {apsm }}$ can be reconstructed by using the first maximal singular value, i.e.

$$
\hat{\mathbf{Y}}_{\mathrm{apsm}}=\sigma_{1} \mathbf{u}_{1} \mathbf{v}_{1}^{\mathrm{T}}
$$

Finally, the reconstructed signal $\hat{\mathbf{s}}$ is extracted by the inverse process of $\hat{\mathbf{Y}}_{\text {apsm }}$ in Eq.14.

The process of the Adaptive Periodic Segment Matrix based on SVD

All the process has been shown in Fig.8. Firstly, the measured signal has been taken into the signal pre-processing. The input signals have been calculated with envelope 
spectrum for selecting the average period length. Then the $\mathrm{R}$ wave peaks and their positions coordinates have been found by using RR intervals constraint. After this, the trajectory matrix $\mathbf{Y}_{\text {apsm }}$ is also formed based on matrix $\mathbf{B}$ in Eq.13 and process to SVD to find out the rank of the matrix equals to one. Then the signal will be recovered by the first maximal singular value.

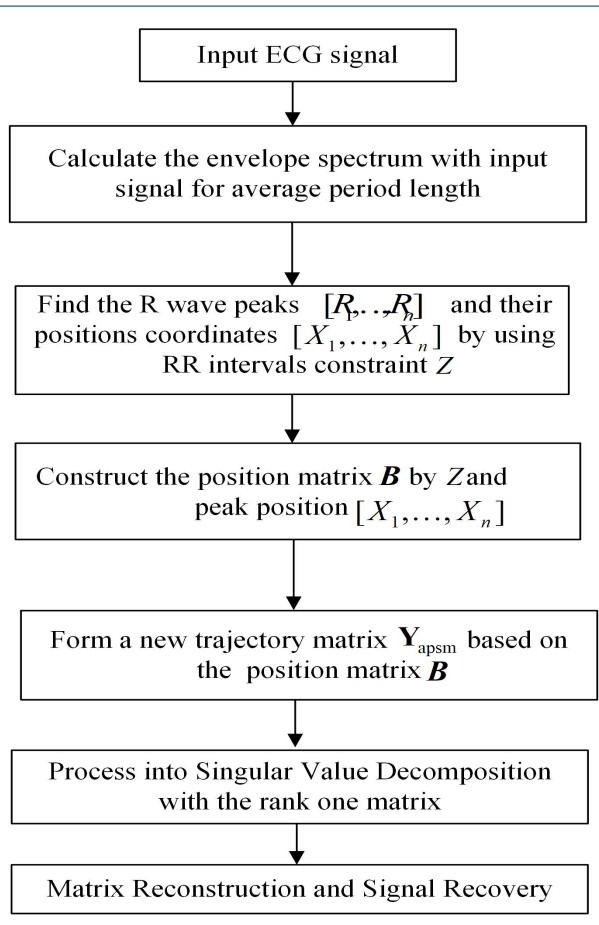

Figure 8 The process of Adaptive Periodic Segment Matrix based on Singular Value Decomposition (1)Convert the frequency of maximum Spectral line as average period length(2) use the RR intervals constraint for R wave peaks selection (3) Position matrix $\mathbf{B}$ can be formed based on R wave peaks and the RR intervals constraint(4) the trajectory matrix can be strict rank one matrix due to B.(5) The first maximal singular value will be used as the signal recovery.

\section{Abbreviation}

ECG: Electrocardiogram; EMG: Electromyogram; APSM: Adaptive Periodic Segment Matrix; SVD: Singular Value Decomposition; $P R D_{1}$ : Zero-mean percent root-mean-square difference; $\mathrm{SNR}_{\text {output }}$ : Output signal to noise ratio; $\mathrm{SNR}_{\text {input }}$ : Input signal to noise ratio; PSM: Periodic Segment Matrix; EMD: Empirical Mode Decomposition; IMF: Intrinsic mode function; SVR: Singular value ratio; EEMD: Ensemble Empirical Mode Decomposition; DWT: Discrete Wavelet Transform; SSA: Singular Spectrum Analysis; LBBB: Left bundle branch block; STEMI: ST elevation myocardial infarction.

Ethics approval and consent to participate Not applicable

Consent for publication

All authors have expressed their consent for this publication.

Availability of data and material

The datasets generated during and/or analysed during the current study are available in the [MIT-BIH Arrhythmia Database] repository, [https://physionet.org/physiobank/database/mitdb/]

Competing interests

The authors declare that they have no competing interests.

Funding

These work are supported by the National Natural Science Foundation of China (No.51905453), China National Key Research and Development Plan for Advanced Rail Transit (No.2016YFB1200401-102) and National Key R\&D Program of China for comprehensive comfort research based on the information and physical space interaction fusion in human-machine-loop (No.2017YFB1201103). 
Author's contributions

Xieqi Chen wrote the whole manuscript and data processing, Jianhui lin gave us some support on academic. And Chenguang Huang provided the concept of this manuscript. Yan Huang has formed the structure of this manuscript. All authors read and approved the final manuscript. Liu He gives the suggestions and helps on theory descriptions and the structure of proposed method.All authors read and approved the final manuscript.

\section{Acknowledgements}

The works were supported by the State key laboratory of traction power. We also thank to our groups for other supports.

\section{Author details}

${ }^{1}$ State key laboratory of traction power, Southwest Jiaotong University, No. 111, North 1st Section, 2nd Ring Road, Chengdu, China. ${ }^{2}$ Weichai Power Co.,Ltd., 197 a, Fushou East Street, high tech Zone, Weifang, China.

\section{References}

1. Kleiger, R.E., Stein, P.K., Bigger, J.T.: Heart Rate Variability: Measurement and Clinical Utility. Annals of Noninvasive Electrocardiology 10(1), 88-101 (2005). doi:10.1111/j.1542-474X.2005.10101.x. Accessed 2019-08-27

2. Lee, C.M., Zhang, Y.T.: Reduction of motion artifacts from photoplethysmographic recordings using a wavelet denoising approach. In: IEEE EMBS Asian-Pacific Conference on Biomedical Engineering, 2003., pp. 194-195. IEEE, Kyoto, Japan (2003). doi:10.1109/APBME.2003.1302650. http://ieeexplore.iee.org/document/1302650/ Accessed 2019-08-27

3. Cuomo, S., De Pietro, G., Farina, R., Galletti, A., Sannino, G.: A revised scheme for real time ECG Signal denoising based on recursive filtering. Biomedical Signal Processing and Control 27, 134-144 (2016). doi:10.1016/j.bspc.2016.02.007. Accessed 2019-09-23

4. Moody, G.B., Mark, R.G.: QRS morphology representation and noise estimation using the Karhunen-Loeve transform. In: [1989] Proceedings. Computers in Cardiology, pp. 269-272. IEEE Comput. Soc. Press, Jerusalem, Israel (1990). doi:10.1109/CIC.1989.130540. http://ieeexplore.iee.org/document/130540/ Accessed 2019-09-24

5. Thakor, N.V., Zhu, Y.-: Applications of adaptive filtering to ECG analysis: noise cancellation and arrhythmia detection. IEEE Transactions on Biomedical Engineering 38(8), 785-794 (1991). doi:10.1109/10.83591

6. Clifford, G., Tarassenko, L., Townsend, N.: One-pass training of optimal architecture auto-associative neural network for detecting ectopic beats. Electronics Letters 37(18), 1126 (2001). doi:10.1049/el:20010762. Accessed 2019-09-24

7. Martis, R.J., Acharya, U.R., Min, L.C.: ECG beat classification using PCA, LDA, ICA and Discrete Wavelet Transform. Biomedical Signal Processing and Control 8(5), 437-448 (2013). doi:10.1016/j.bspc.2013.01.005. Accessed 2019-11-16

8. Pinto, V.d.: Filters for the reduction of baseline wander and muscle artifact in the ECG. Journal of Electrocardiology 25, 40-48 (1992). doi:10.1016/0022-0736(92)90060-D

9. van Alsté, J.A., van Eck, W., Herrmann, O.E.: ECG baseline wander reduction using linear phase filters. Computers and Biomedical Research 19(5), 417-427 (1986). doi:10.1016/0010-4809(86)90037-6. Accessed 2019-11-16

10. Lulu, Z., Lin, X., Yuliang, Y.: Removal of baseline drift from ECG signals based on morphology and adaptive filter. In: Proceedings of the 31st Chinese Control Conference, pp. 3750-3753 (2012)

11. Rakshit, M., Das, S.: An efficient ECG denoising methodology using empirical mode decomposition and adaptive switching mean filter. Biomedical Signal Processing and Control 40, 140-148 (2018). doi:10.1016/j.bspc.2017.09.020. Accessed 2019-09-23

12. Sharma, R.R., Pachori, R.B.: Baseline wander and power line interference removal from ECG signals using eigenvalue decomposition. Biomedical Signal Processing and Control 45, 33-49 (2018). doi:10.1016/j.bspc.2018.05.002. Accessed 2019-11-16

13. Dora, C., Biswal, P.K.: Correlation-based ECG Artifact Correction from Single Channel EEG using Modified Variational Mode Decomposition. Computer Methods and Programs in Biomedicine 183, 105092 (2020). doi:10.1016/j.cmpb.2019.105092. Accessed 2019-11-16

14. Agrawal, S., Gupta, A.: Fractal and EMD based removal of baseline wander and powerline interference from ECG signals. Computers in Biology and Medicine 43(11), 1889-1899 (2013). doi:10.1016/j.compbiomed.2013.07.030. Accessed 2019-11-13

15. Bharath, H.N., Prabhu, K.M.M.: A new LMS based adaptive interference canceller for ECG power line removal. In: 2012 International Conference on Biomedical Engineering, ICoBE 2012, pp. 68-73 (2012). doi:10.1109/ICoBE.2012.6178957

16. Zhao, Z., Chen, Y.: A New Method for Removal of Baseline Wander and Power Line Interference in ECG Signals. In: 2006 International Conference on Machine Learning and Cybernetics, pp. $4342-4347$ (2006). doi:10.1109/ICMLC.2006.259082

17. Hamilton, P.S.: A comparison of adaptive and nonadaptive filters for reduction of power line interference in the ECG. IEEE Transactions on Biomedical Engineering 43(1), 105-109 (1996). doi:10.1109/10.477707. Accessed 2019-11-22

18. Taralunga, D.D., Gussi, I., Strungaru, R.: Fetal ECG enhancement: Adaptive power line interference cancellation based on Hilbert Huang Transform. Biomedical Signal Processing and Control 19, 77-84 (2015) doi:10.1016/j.bspc.2015.03.009

19. Marouf, M., Saranovac, L., Vukomanovic, G.: Algorithm for EMG noise level approximation in ECG signals. Biomedical Signal Processing and Control 34, 158-165 (2017). doi:10.1016/j.bspc.2017.02.002. Accessed 2019-11-16

20. Singh, G.: Removal of EMG Interference from Electrocardiogram Using Back Propagation 1(6), 6 (2007) 
21. Chang, K.-M., Liu, S.-H.: Gaussian Noise Filtering from ECG by Wiener Filter and Ensemble Empirical Mode Decomposition. Journal of Signal Processing Systems 64(2), 249-264 (2011). doi:10.1007/s11265-009-0447-z. Accessed 2019-08-27

22. Miljković, N., Popović, N., Djordjević, O., Konstantinović, L., Šekara, T.B.: ECG artifact cancellation in surface EMG signals by fractional order calculus application. Computer Methods and Programs in Biomedicine 140, 259-264 (2017). doi:10.1016/j.cmpb.2016.12.017. Accessed 2019-11-29

23. Poornachandra, S.: Wavelet-based denoising using subband dependent threshold for ECG signals. Digital Signal Processing 18(1), 49-55 (2008). doi:10.1016/j.dsp.2007.09.006. Accessed 2019-08-27

24. Blanco-Velasco, M., Weng, B., Barner, K.E.: ECG signal denoising and baseline wander correction based on the empirical mode decomposition. Computers in Biology and Medicine 38(1), 1-13 (2008). doi:10.1016/j.compbiomed.2007.06.003. Accessed 2019-08-27

25. Castro, B., Kogan, D., Geva, A.B.: ECG feature extraction using optimal mother wavelet. In: 21st IEEE Convention of the Electrical and Electronic Engineers in Israel. Proceedings (Cat. No.00EX377), pp. 346-350. IEEE, Tel-Aviv, Israel (2000). doi:10.1109/EEEI.2000.924422. http://ieeexplore.iee.org/document/924422/ Accessed 2019-08-28

26. Mortezaee, M., Mortezaie, Z., Abolghasemi, V.: An Improved SSA-Based Technique for EMG Removal from ECG. IRBM 40(1), 62-68 (2019). doi:10.1016/j.irbm.2018.11.004. Accessed 2019-08-27

27. Ahmed, S.M., Al-Zoubi, Q., Abo-Zahhad, M.: A hybrid ECG compression algorithm based on singular value decomposition and discrete wavelet transform. Journal of Medical Engineering \& Technology 31(1), 54-61 (2007). doi:10.1080/03091900500518811

28. Nougarou, F., Massicotte, D., Descarreaux, M.: Efficient procedure to remove ECG from sEMG with limited deteriorations: Extraction, quasi-periodic detection and cancellation. Biomedical Signal Processing and Control 39, 1-10 (2018). doi:10.1016/j.bspc.2017.07.019. Accessed 2019-11-29

29. Kanjilal, P.P., Palit, S., Saha, G.: Fetal ECG extraction from single-channel maternal ECG using singular value decomposition. IEEE Transactions on Biomedical Engineering 44(1), 51-59 (1997). doi:10.1109/10.553712. Accessed 2019-08-27

30. Huang, C., Lin, J., Ding, J., Huang, Y.: A Novel Wheelset Bearing Fault Diagnosis Method Integrated CEEMDAN, Periodic Segment Matrix, and SVD. Shock and Vibration 2018, 1-18 (2018). doi:10.1155/2018/1382726. Accessed 2019-08-29

31. Kanjilal, P.P., Palit, S.: On multiple pattern extraction using singular value decomposition. IEEE Transactions on Signal Processing 43(6), 1536-1540 (1995). doi:10.1109/78.388873. Accessed 2019-08-27

32. The MIT-BIH Arrhythmia Database. http://physionet.org/physiobank/database/mitdb/

33. Vautard, R., Yiou, P., Ghil, M.: Singular-spectrum analysis: A toolkit for short, noisy chaotic signals. Physica D: Nonlinear Phenomena 58(1), 95-126 (1992). doi:10.1016/0167-2789(92)90103-T

34. Vautard, R., Ghil, M.: Singular spectrum analysis in nonlinear dynamics, with applications to paleoclimatic time series. Physica D: Nonlinear Phenomena 35(3), 395-424 (1989). doi:10.1016/0167-2789(89)90077-8

35. Golafshan, R., Yuce Sanliturk, K.: SVD and Hankel matrix based de-noising approach for ball bearing fault detection and its assessment using artificial faults. Mechanical Systems and Signal Processing 70-71, 36-50 (2016). doi:10.1016/j.ymssp.2015.08.012. Accessed 2019-12-03

36. Jiang, H., Chen, J., Dong, G., Liu, T., Chen, G.: Study on Hankel matrix-based SVD and its application in rolling element bearing fault diagnosis. Mechanical Systems and Signal Processing 52-53, 338-359 (2015). doi:10.1016/j.ymssp.2014.07.019. Accessed 2019-12-03

37. The MIT-BIH Noise Stress Test Database. http://physionet.org/physiobank/database/nstdb/

38. Abo-Zahhad, M., Al-Ajlouni, A.F., Ahmed, S.M., Schilling, R.J.: A new algorithm for the compression of ECG signals based on mother wavelet parameterization and best-threshold levels selection. Digital Signal Processing 23(3), 1002-1011 (2013). doi:10.1016/j.dsp.2012.11.005. Accessed 2019-11-16

39. Agrawal, S., Gupta, A.: Projection operator based removal of baseline wander noise from ECG signals. In: 2013 Asilomar Conference on Signals, Systems and Computers, pp. 957-961. IEEE, Pacific Grove, CA, USA (2013). doi:10.1109/ACSSC.2013.6810431. http://ieeexplore.ieee.org/document/6810431/ Accessed 2019-11-27

40. Ibanez, B., James, S., Agewall, S., Antunes, M.J., Bucciarelli-Ducci: 2017 ESC Guidelines for the management of acute myocardial infarction in patients presenting with ST-segment elevation. European Heart Journal 39(2), 119-177 (2018). doi:10.1093/eurheartj/eh×393. Accessed 2019-11-28 
Figures
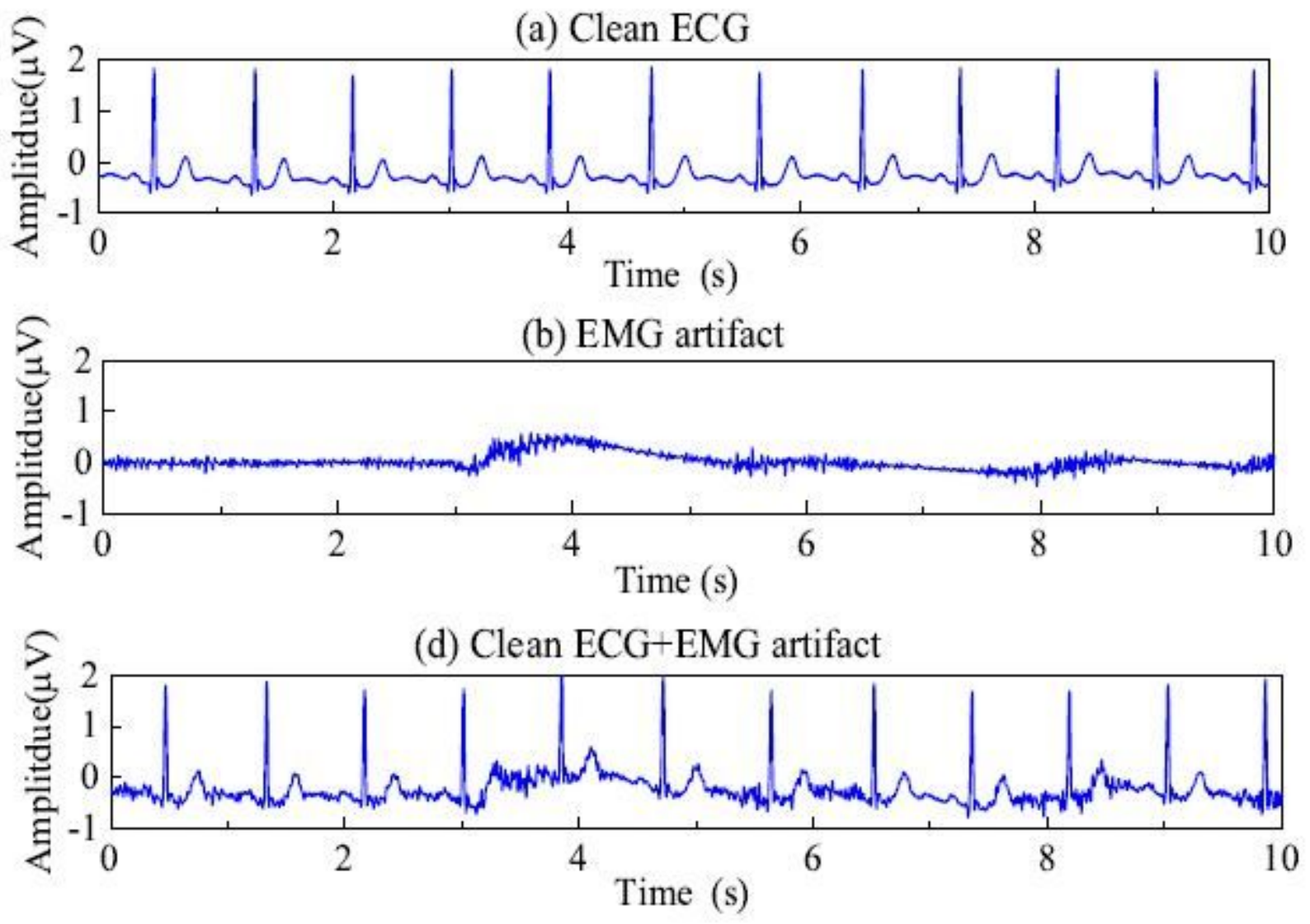

\section{Figure 1}

The constructed simulated signals with clean ECG and EMG artifact (a)Clean ECG (b)EMG artifact (c)Clean ECG+EMG artifact 


\section{Input ECG signal}

Calculate the envelope spectrum with input signal for average period length

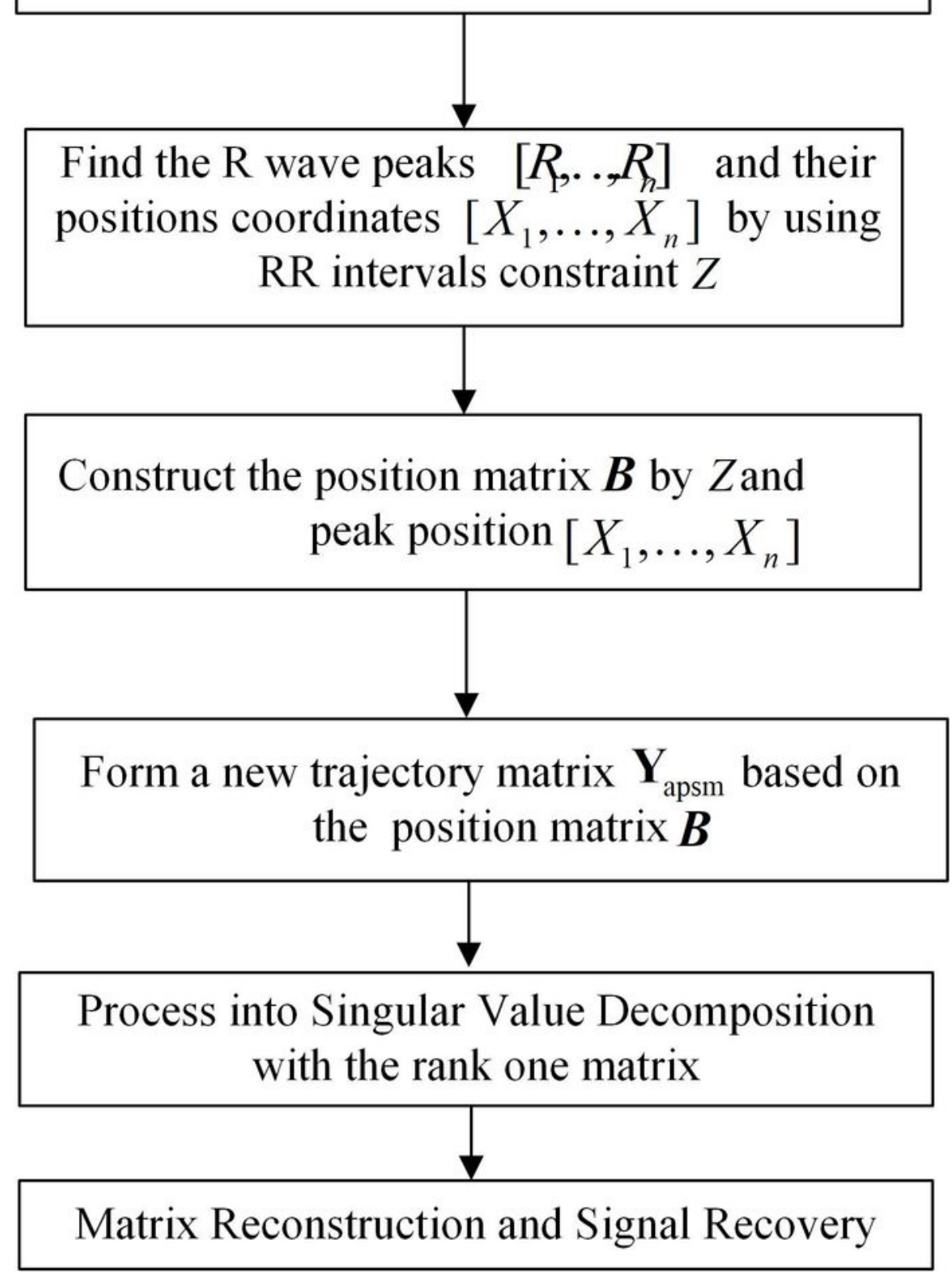

Figure 2

The comparative performance SNRoutput of the proposed APSM-SVD with other methods over 5 segments 

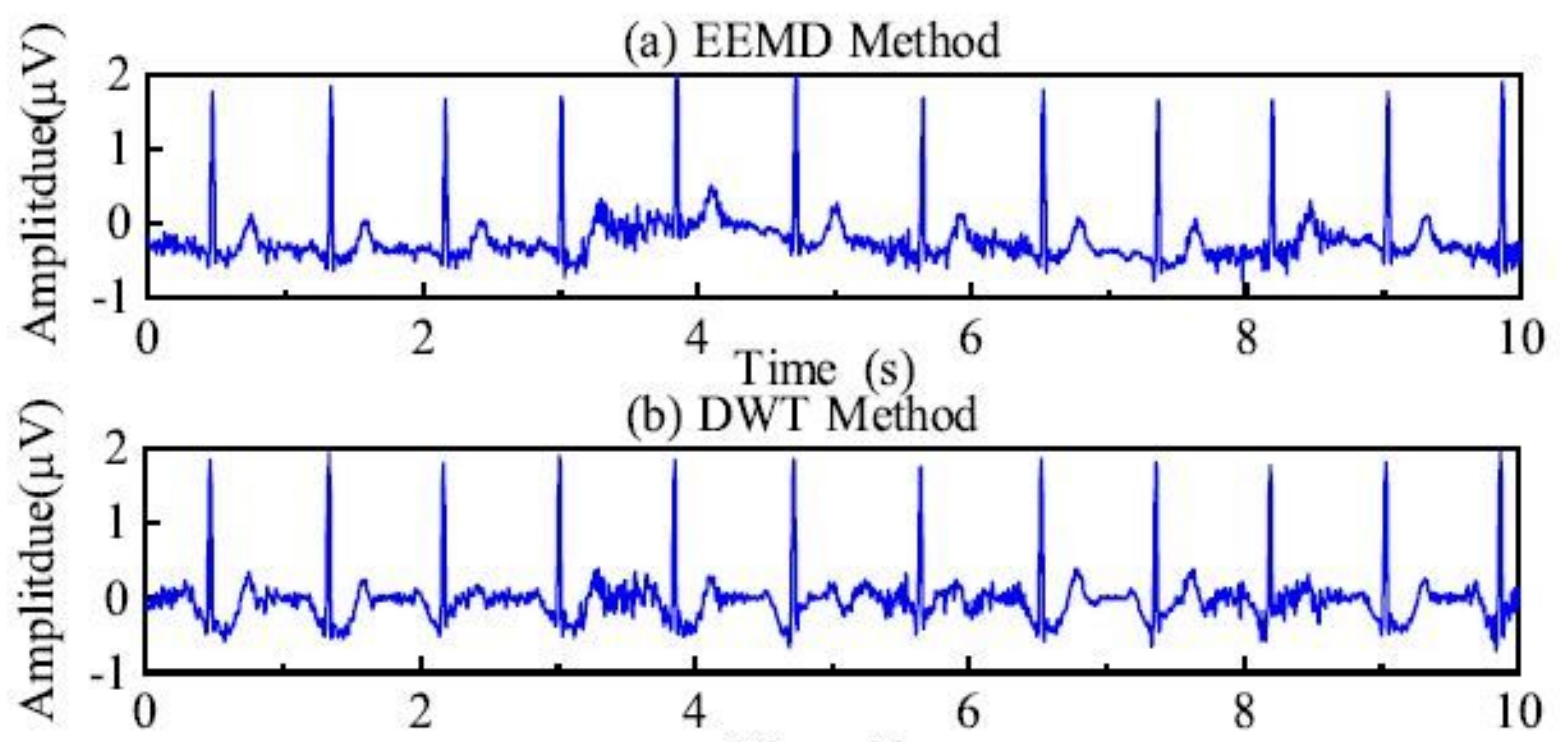

Time (s)
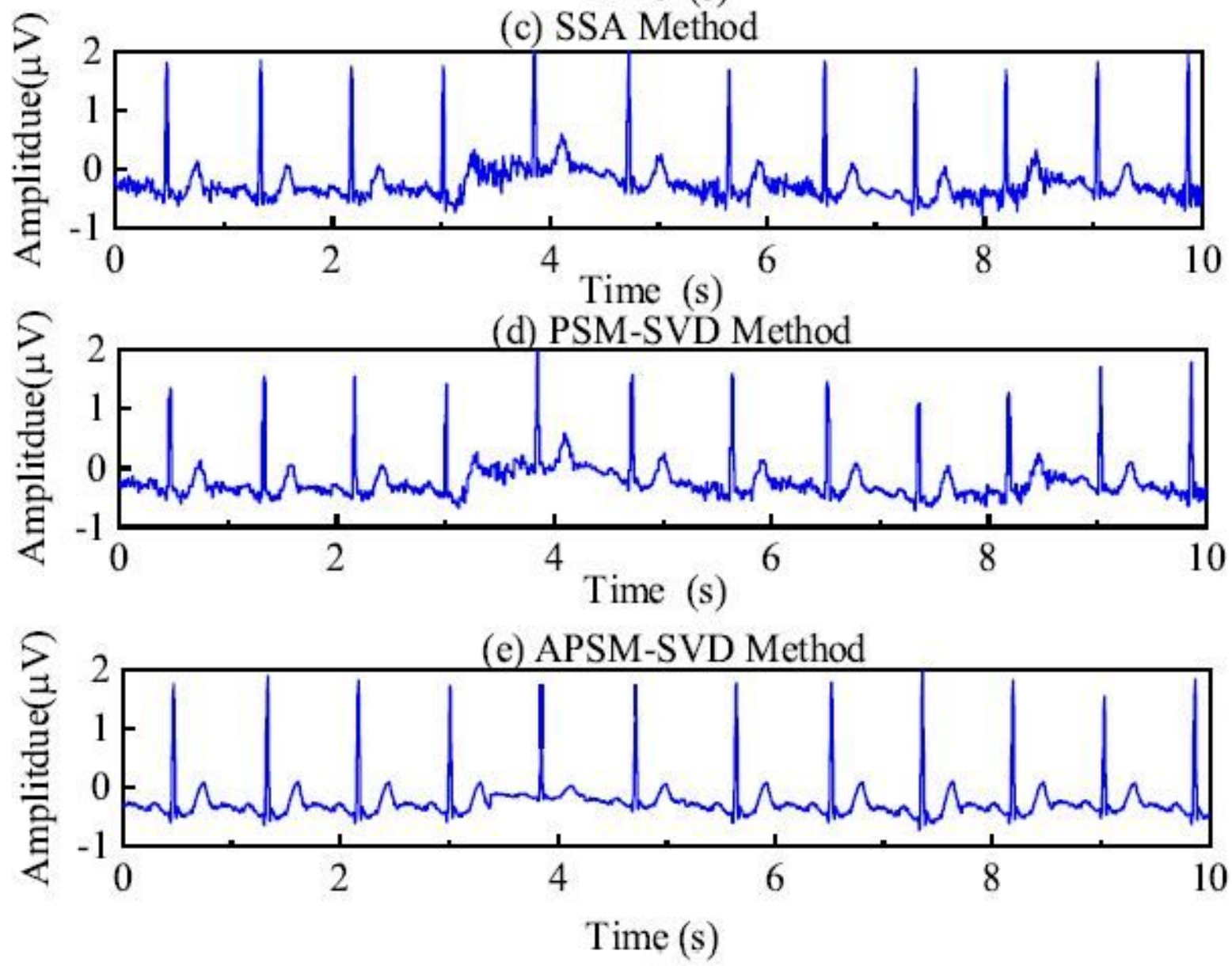

Figure 3

Reconstructed ECG signals from noisy ECG record by using the proposed APSM-SVD, EEMD, DWT, PSMSVD and SSA(a)EEMD (b)DWT (c)SSA (d)PSM-SVD (e)APSM-SVD 


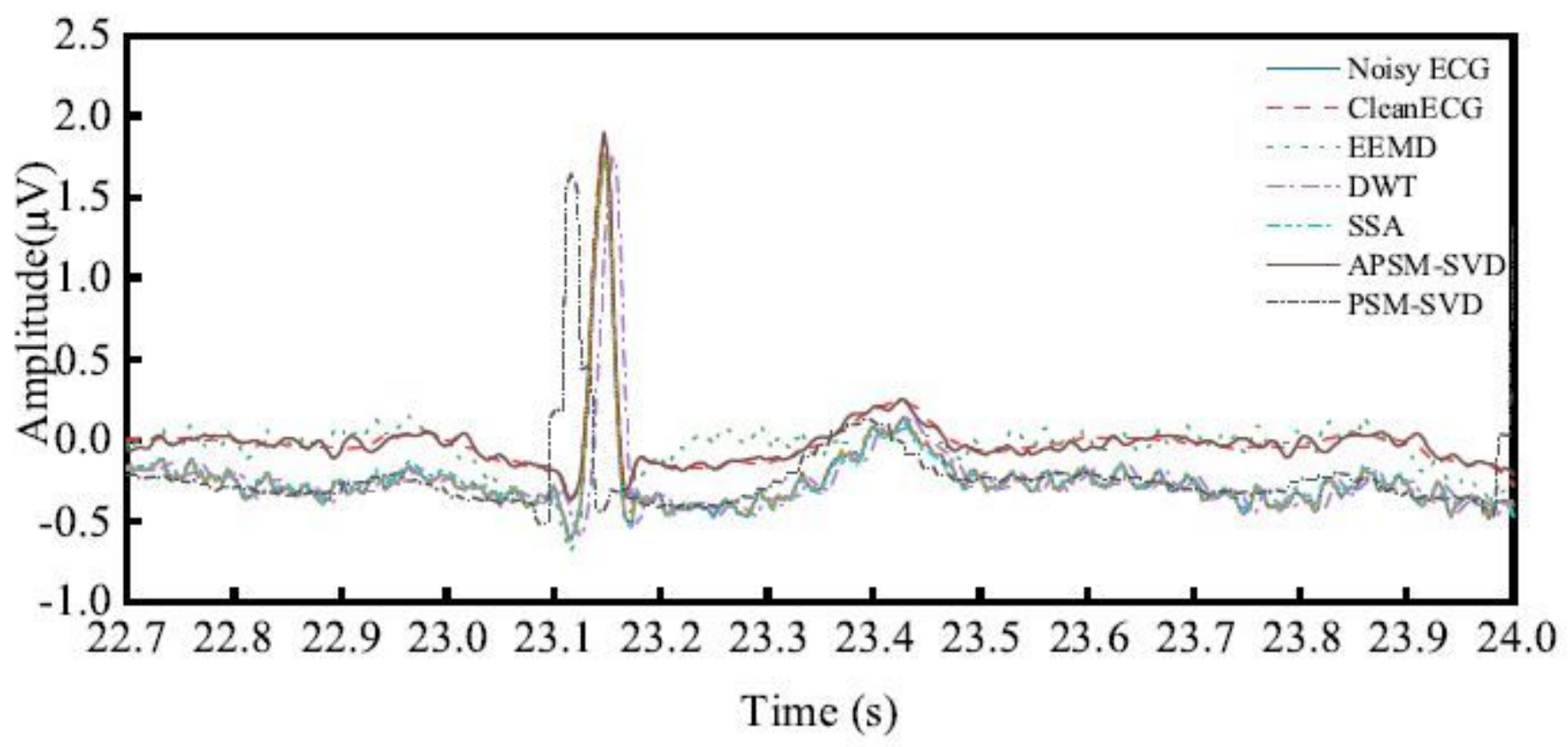

(a) The amplitude of EMG noise is relatively small

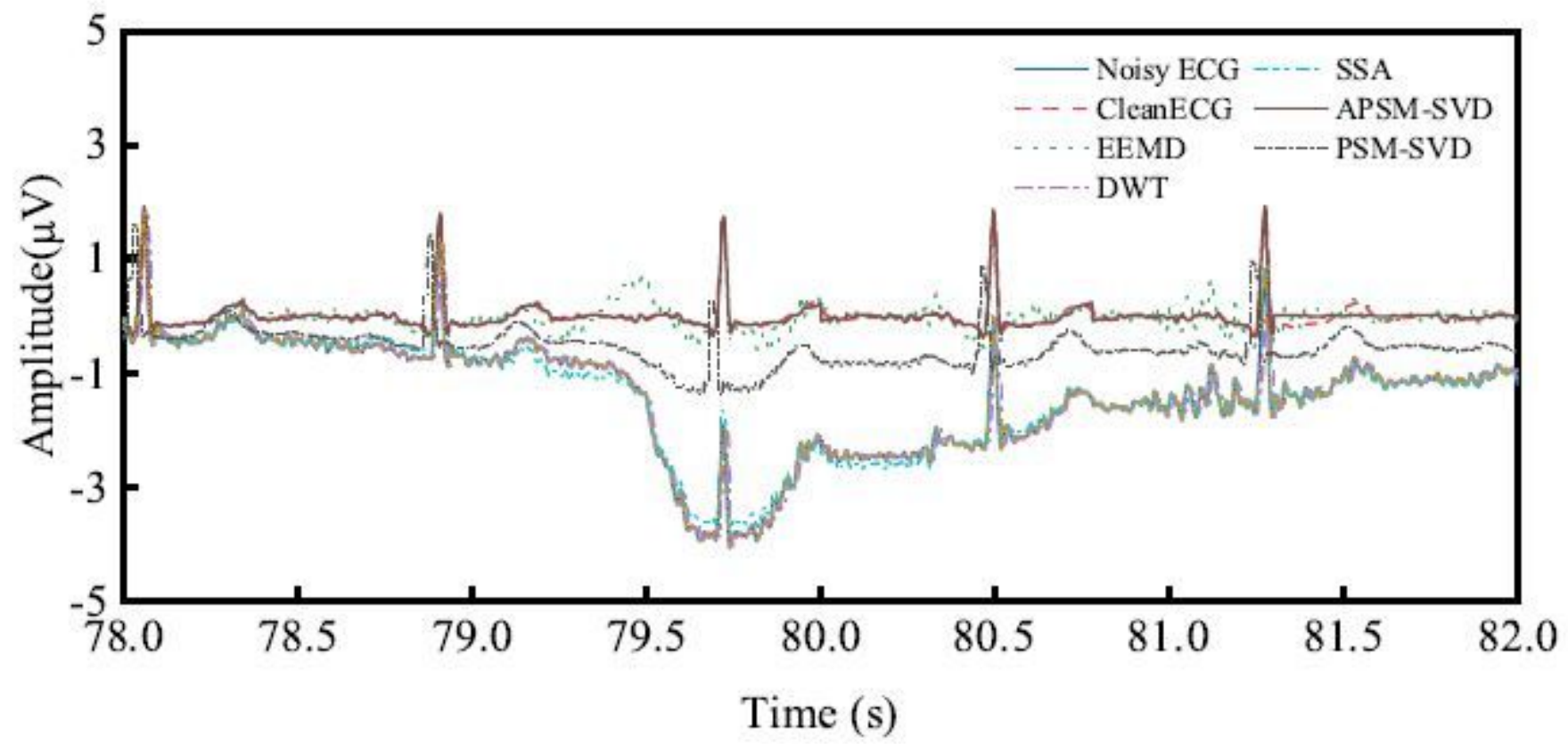

(b) The amplitude of EMG noise is relatively larger

Figure 4

The reconstructed signals obtained from the proposed technique compared to EMD, DWT, SSA and PSMSVD for QRS complex in 103 record.(a) The amplitude of EMG noise is smaller (b)The amplitude of EMG noise is larger 

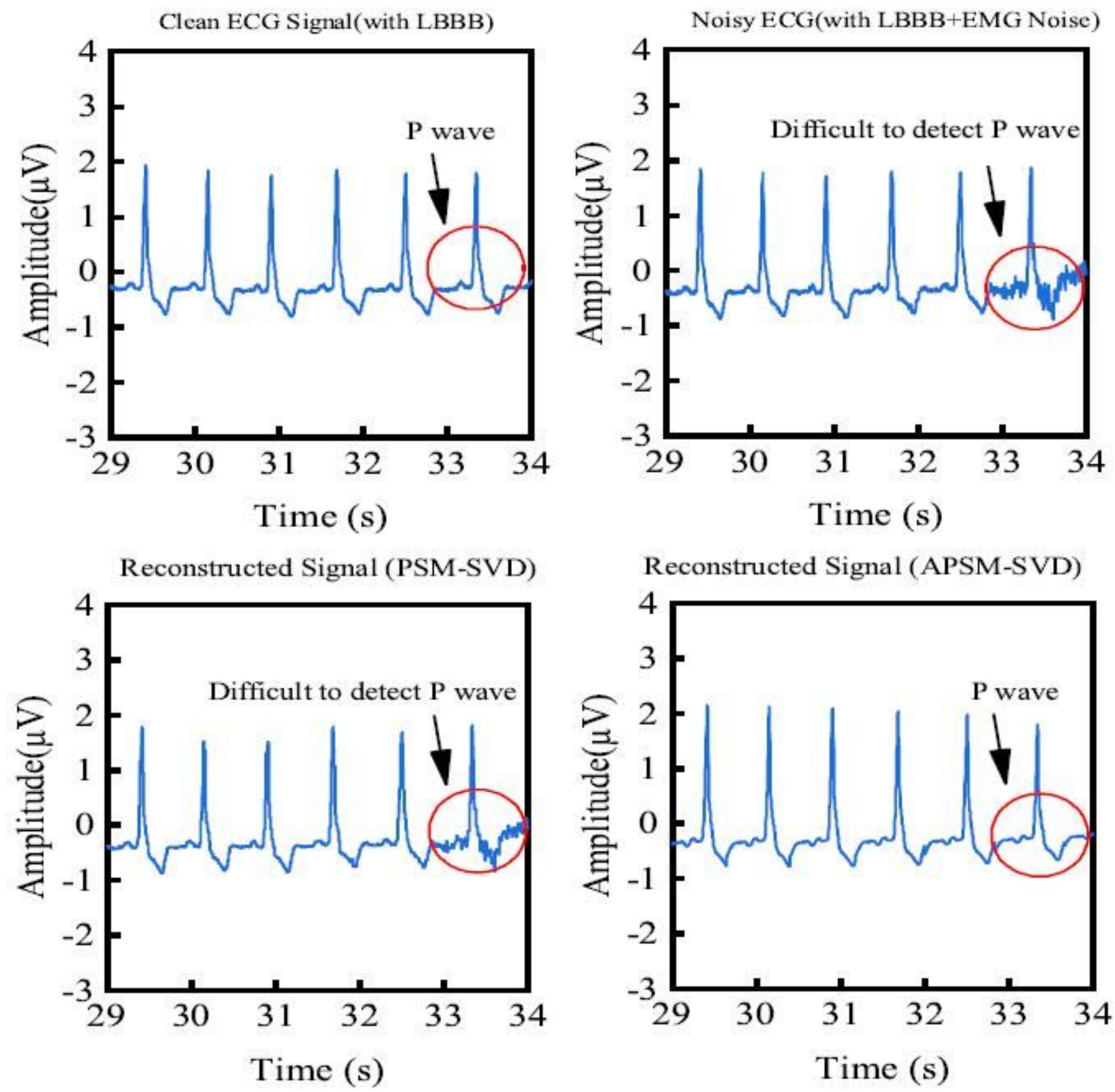

Figure 5

The detection of the R peak, $\mathrm{P}$ wave and QRS complex in 214 LBBB record 

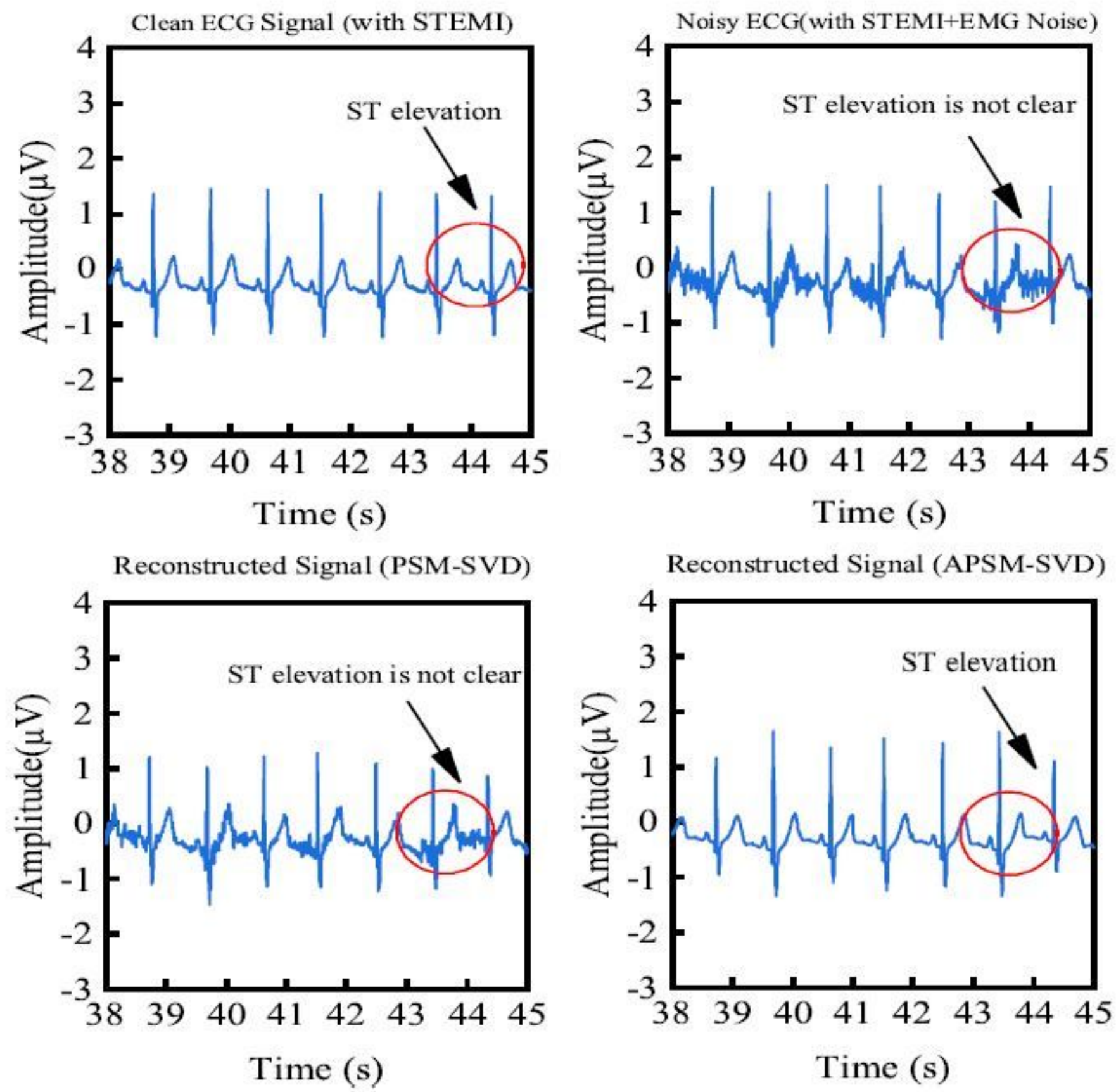

Figure 6

The detection of the R peak, ST elevation and QRS complex in 231 STEMI record 
(a) The first 3 rows of trajectory matrix in APSM

(b) The first 3 rows of trajectory matrix in PSM
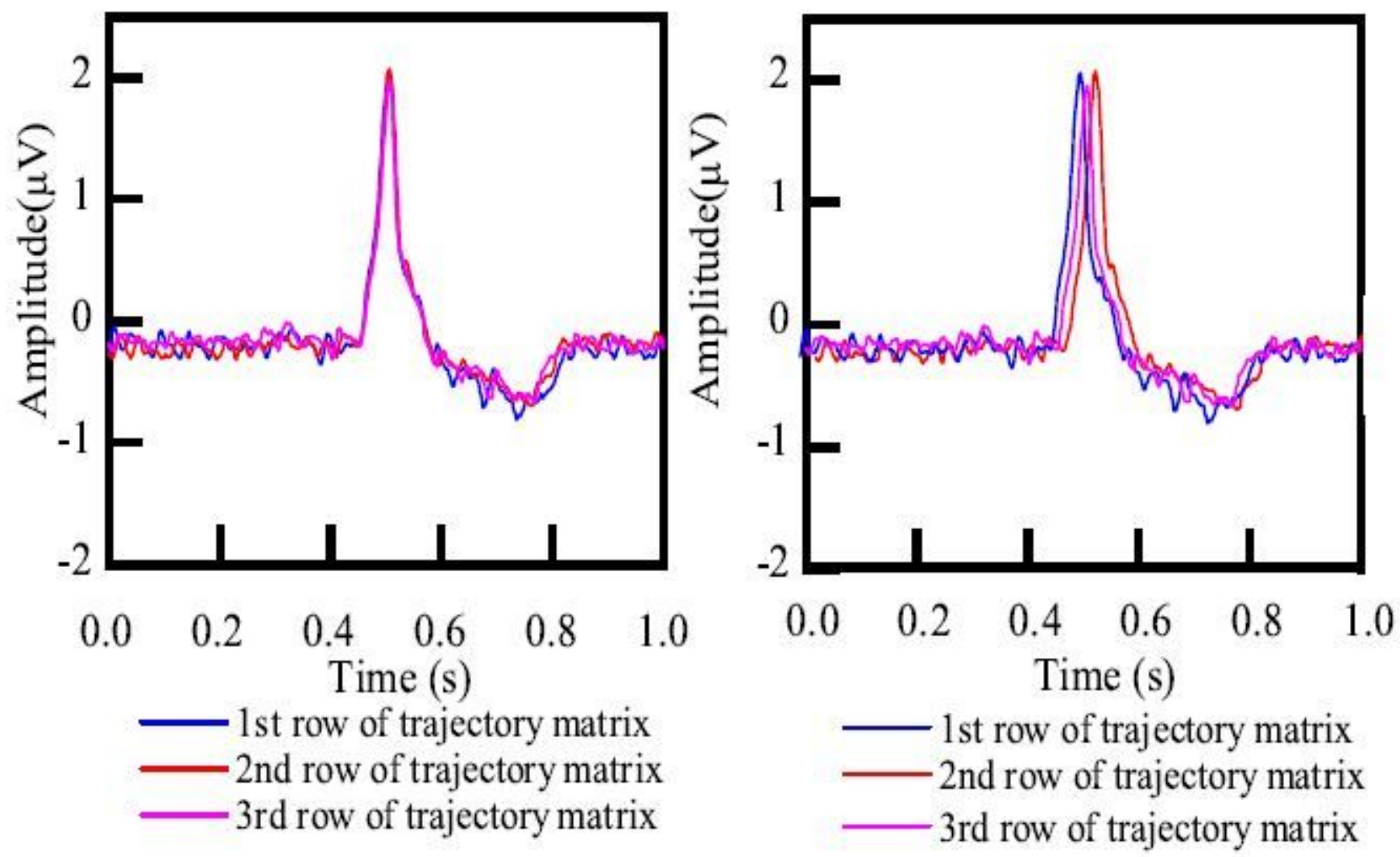

Figure 7

The comparison of first three rows in APSM and PSM (a) The first 3 rows in APSM (b) The first 3 rows in PSM 


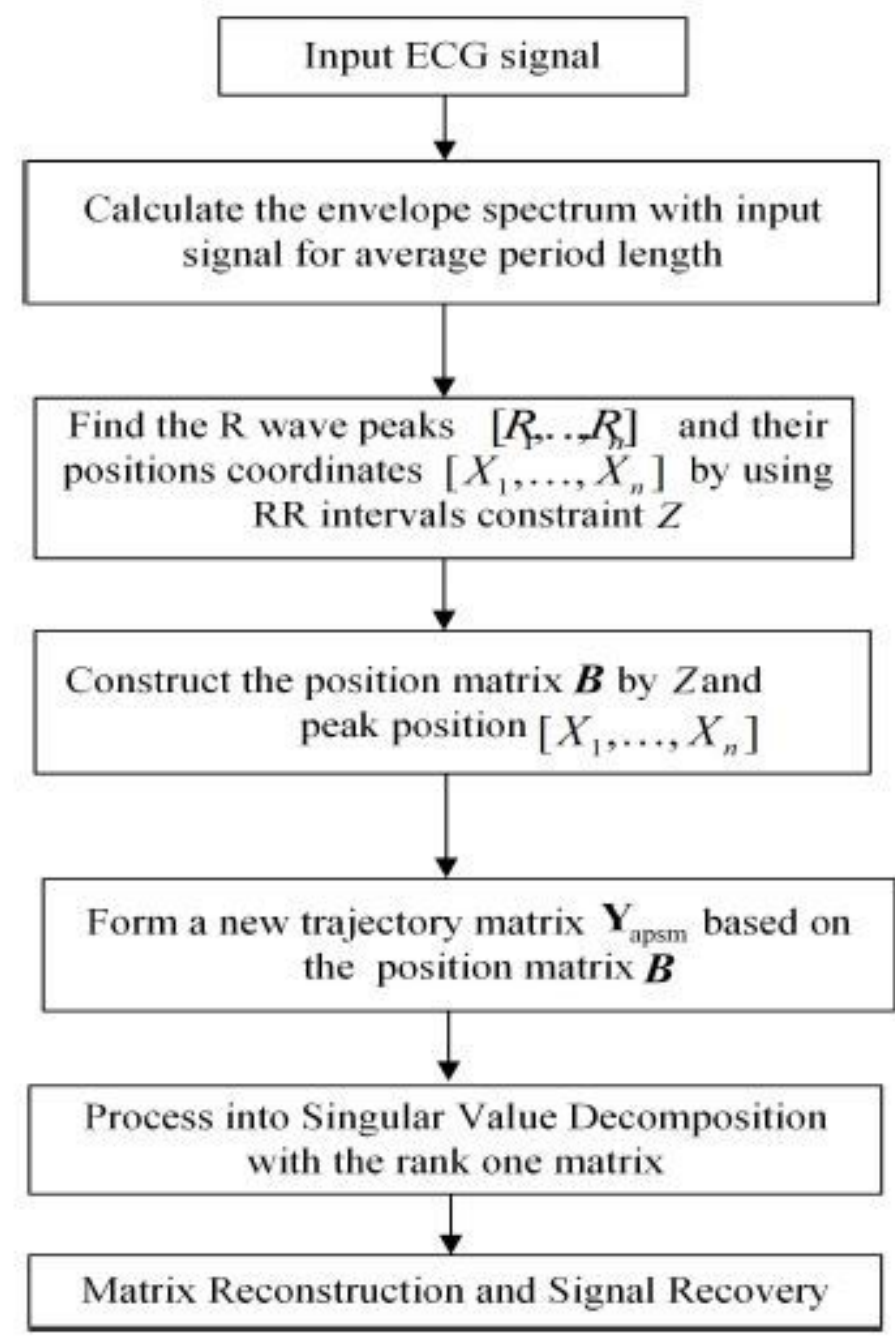

\section{Figure 8}

The process of Adaptive Periodic Segment Matrix based on Singular Value Decomposition (1)Convert the frequency of maximum Spectral line as average period length(2) use the RR intervals constraint for $R$ wave peaks selection (3) Position matrix B can be formed based on R wave peaks and the RR intervals constraint(4) the trajectory matrix can be strict rank one matrix due to B.(5) The first maximal singular value will be used as the signal recovery. 